To appear in Transportmetrica A: Transport Science

Vol. 00, No. 00, Month 20XX, 1-31

\title{
MANUSCRIPT
}

\section{A Representation of Partial Spatial Knowledge: A Cognitive Map Approach for Evacuation Simulations}

\author{
Erik Andresen*, Mohcine Chraibi and Armin Seyfried \\ Jülich Supercomputing Centre, Forschungszentrum Jülich GmbH \\ (May 2016, revised Januar 2017, revised again November 2017)
}

\begin{abstract}
Usually, routing models in evacuation simulations assume that agents have comprehensive and global knowledge about the building's structure. They neglect the fact that pedestrians might possess no or only parts of information about their position relative to final exits and possible routes leading to them. For the sake of a more realistic description of the routing process, we introduce the systematics of using partial spatial knowledge. Particularly, we present an agent-based approach modeling the inaccurate mental representation of pedestrians' spatial knowledge (the cognitive map). In addition, the model considers further principles and constraints of human wayfinding.

Furthermore, we present results of a field study we conducted in an office building. The purpose of this study was to investigate route choices of people in dependency on their familiarity with the building. Our modeling approach is then calibrated using the obtained results. In this context, the distribution of routes which were used by the subjects are compared with results of the model.
\end{abstract}

Keywords: Route Choice; Evacuation Simulations; Wayfinding; Cognitive Map; Partial Knowledge Representation;

\section{Introduction}

As almost all fundamental abilities of our species (e.g. to communicate, visit, merchandise, hunt, etc.) rely on effective and efficient travel strategies, one could say that wayfinding is an ubiquitous process people have to deal with on a daily basis. Although the human being is daily faced with wayfinding issues, many of the processes within the wayfinding procedure remain obscure. Tasks such as driving to the workplace, for instance, are processed automatically within the brain without the necessity of explicit thoughts (?).

A majority of mechanisms and aspects involved in the wayfinding procedure are not entirely known. Particularly, the question of how the various tools, strategies or mechanisms work together in wayfinding tasks is not answered completely. Researchers from several fields, for example neuroscience, cognitive science, computer science, geography, architecture, psychology, etc. are contributing to the wayfinding topic investigating individual aspects and their collaboration.

Considering human wayfinding abilities is important in evacuation simulations. It has been shown that, in certain scenarios, the pedestrians' exit choices and route planning can decisively influence the simulated evacuation progress and therefore the predicted evacuation time (?). In many pedestrian simulation frameworks the routes that simulated persons take are determined by shortest path or travel time optimization algorithms 
in advance. This might be an acceptable and appropriate approach for the simulation of scenarios in which wayfinding is trivial. However, particularly in complex buildings, pedestrians' knowledge degrees about their environment normally vary and are unlikely to be complete nor global. The assumption that all pedestrians are provided with comprehensive global knowledge about the building's structure is a rough approximation, for example when the majority of pedestrians are first time visitors of a facility. Even less, they are able to evaluate metric information about various routes accurately to identify the shortest one. In fact, the knowledge status of a group of pedestrians vary according to the number of visits and the capability to learn the spatial structure of new environments $(?)$.

In this paper, we introduce new modeling approaches which provide simulated pedestrians with a differing, partial and inaccurate spatial knowledge representation, the so-called cognitive map (see Sec. 4.4). Furthermore, the model considers the implementation of action-based representations (see Sec. 4.5) and generalized knowledge (see Sec. 2.4.3). It is explained in detail how the agents use their spatial knowledge and select a visible intermediate destination to proceed their way to their main destination.

We present results of a field study which we conducted to investigate the route choices of people in dependency on their spatial knowledge about the area of the study. These results are used to calibrate the cognitive map model. Although the calibrated model is capable of reproducing the resulting route choice distributions from the study approximately, it is not claimed to predict a route choice of an individual in an arbitrary situation. The route choice of an individual may depend on a huge number of influencing factors. This paper focus on selected factors in a simplified and generalized way. The presented approach do not claim to predict individual route choices exactly. The model only provides an educated guess of a route choice distribution of a group of pedestrians in which a majority relies on considered strategies and personal habits of individuals play a minor role. Even in the best case, this educated guess is only an approximation of a route choice distribution measured in a real system.

This paper is structured as follows. Section 2 comprises a literature review on aspects, principles, used tools and known strategies of wayfinding. The presented findings from the literature are relevant for this paper as they were considered for the development of the modeling approaches. The literature review also incorporates previously developed route choice models for pedestrian simulations that consider human wayfinding abilities (see Sec. 2.5). To provide an overview of the tools and strategies involved in wayfinding tasks during evacuation, a new wayfinding taxonomy based on evidence and taxonomies from the literature is introduced (see Sec. 3). In Section 4 the above mentioned modeling approaches are explained. Various example cases are presented that explain the model's functionality in detail (see Sec. 4.8). In Section 5 the results of the field study are presented, discussed, and used to calibrate and test the cognitive map model.

Finally, we conclude and assess the model's possibilities under consideration of the comparison between results from the model and the field study.

\section{Human wayfinding - Literature review}

\subsection{Navigation - locomotion and wayfinding}

Navigation plays a major role in the human life. People are faced with them every day e.g. while traveling and navigating in a building. Nevertheless, the majority of underlying mental mechanisms involved in the navigation process are still not entirely known.

On the one hand, navigational tasks require locomotive abilities (??). These abilities enable people to move to visible destinations without colliding with walls, obstacles, other 
persons, etc. Therefor, the immediate surrounding is observed by sensory-input systems (?). On the other hand, wayfinding tasks are involved in the process of navigation as well. A wayfinding task comprises the localization of a specific non-visible destination and the planning of an "appropriate" route leading (possibly via intermediate targets) to the final destination (???). As a consequence wayfinding requires the localization of objects or places beyond the visible surrounding.

? describe wayfinding as a four step process. The first step comprises the determination of the own position, whereas the second step consists of the planning or choosing of a suitable route. If no route could be found, one has at least to choose a desired direction. Additionally, effective wayfinding requires the ability to detect parting from the desired route or the desired direction. Finally, the final destination has to be recognized when it is reached (?).

Wayfinding requires the capability to perceive, memorize, retrieve, and assess spatial information in terms of mental representations of the environment. People's abilities to successfully solve wayfinding tasks differ widely (?). Particularly, individual differences occur concerning their capability to encode and process spatial information (?). Qualitative gender differences have been found in the selection of spatial information and strategies aiding the wayfinding process (?).

\subsection{The cognitive map}

The cognitive map depicts the mental representation of someone's large-scale ${ }^{1}$ environment's spatial structure. The representation consists of memorized objects and their spatial relations to each other (??).

Primarily, ? introduced the term cognitive map in the 1930s. He conducted routing experiments on rats. Without being driven by stimuli the rats were able to retrieve the direction leading them to a destination they visited multiple times before. As a consequence ? noticed that the rats hold the ability to memorize the positions of specific places relative to others.

? provided evidence about the fact that the rat's hippocampal formation is mainly involved in the establishment of a reference map system (the cognitive map). The hippocampus stores and retrieves spatial memories by using place cells (?). Specific place cells are triggered when the rat reaches a specific point (?). It has been shown that further cells are collaborating with place cells in building the capability to store, retrieve, and use spatial memories. As related to these, head direction cells (?), grid cells (?), and border cells (?) have to be mentioned. A collaboration of similar cells serving orientation and wayfinding were discovered in the human and various other mammals' brains (??).

The cognitive map enables people to determine the relative location of a non-visible destination beyond their immediate surroundings by processing and evaluating the information in the map. In many cases, cognitive maps of unfamiliar environments are provided with scarce information (?). Even after multiple stays in an environment the related cognitive map may consist of incomplete, inaccurate information (?). The externalization of cognitive maps in the form of sketched drawings shows few similarities with the corresponding physical space (?). The sketched drawings suggest that the cognitive map is rather an inaccurate (abstract) representation of the perceived environment (?). Especially errors concerning the exact metric distances between objects and angles between connections of objects, e.g. turn angles, occur (??). However, the provided information, even if not complete, can be sufficient to solve the wayfinding problem due

\footnotetext{
${ }^{1}$ The large- or medium-scale environment includes also parts of the surroundings which are not currently accessible
} by sensory-input. 
to the fact that many tasks can be solved with the help of topological relations (?). A possible explanation why we leave out several detailed information or rather only store selected parts and topological information of the environment could be the efficiency and the minor requirements for memory that come along with this strategy (?).

\subsection{Landmarks and further components of the cognitive map}

Landmarks are salient points or area objects in the environment. They serve as the main components in the formation of the cognitive map (?). Landmarks are recognized and memorized because of their conspicuousness, uniqueness or rather disparity to other objects (?). Landmarks could be a known place, a great tower, an old looking house or a statue. A view of a set-up of multiple objects or a scene could also be considered as a landmark (??). Specific landmarks serve as anchor points or rather as the origin of the cognitive map's reference frame. Hence, people tend to determine other landmarks' and their own location relative to an anchoring landmark, for instance a high, globally visible tower (?).

In many cases, route segments start and end at landmarks ${ }^{2}$. The selection of proceeding paths or directions are often made at landmarks ${ }^{3}$ (?). By tendency, landmarks are preferred to be internalized and remembered because of their disparity concerning visible shape or structure (?). Nevertheless, objects without optical particularities might also be remembered due to sociocultural or personal relevance (?). A study by ? showed that $50 \%$ of city objects were stated as remembered (landmarks) by almost all participants. The other remembered objects were related to personal interests.

The cognitive map is basically assumed to be the mental representation of landmarks and their spatial relations to each other. Information about the mentioned relations incorporates clues about direct or indirect connections (line or route segments) between landmarks (?).

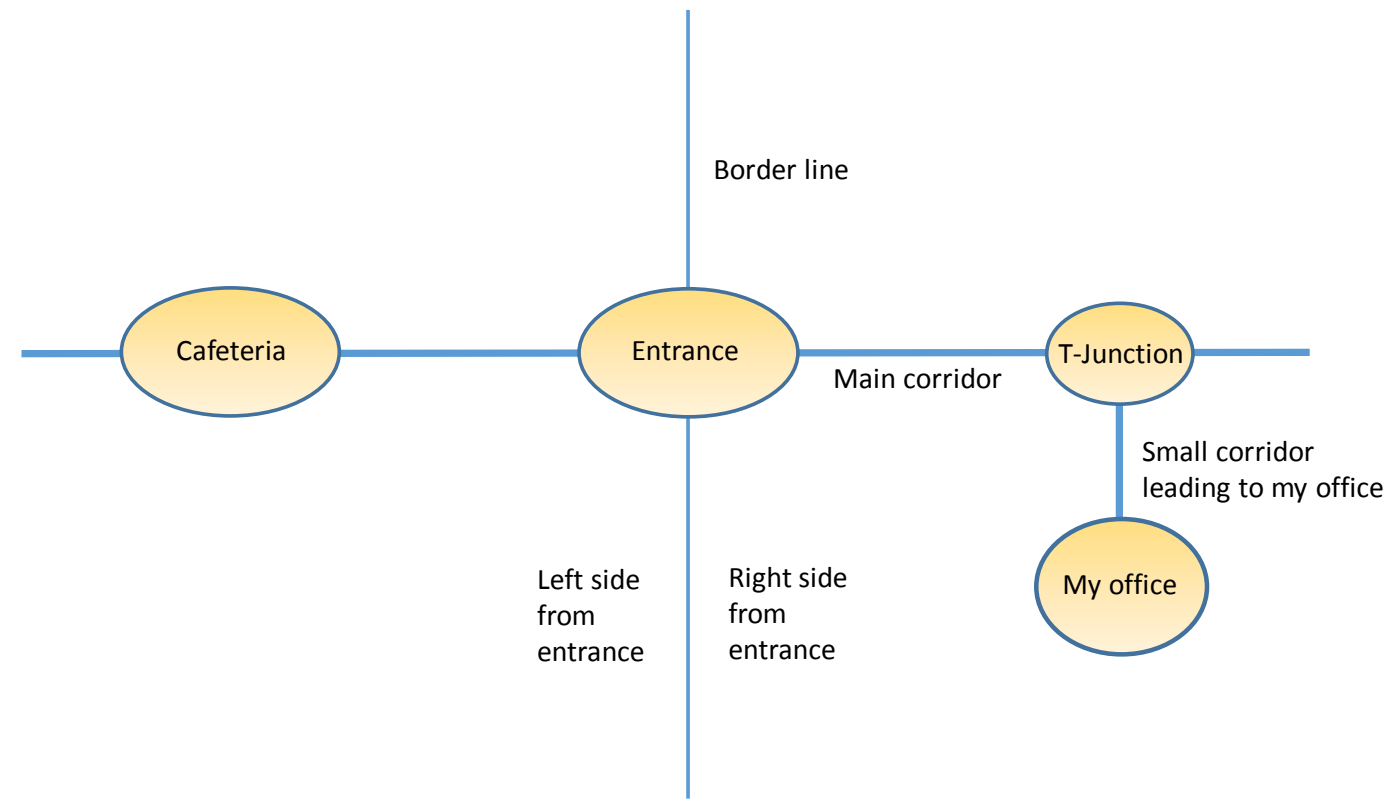

Figure 1. Possible mental representation of a person's workplace.

\footnotetext{
${ }^{2}$ Landmark as origin or destination

${ }^{3}$ Landmark as an onroute choice point
} 
Fig. 1 shows a visualization of a fictional cognitive map and its items. The map does not incorporate any metric information. However, it provides topological information, particularly it shows which objects are directly connected to each other by a walkable path. Furthermore, the wayfinder (owner of the map) is able to infer spatial relations qualitatively such as "starting from the t-junction, the cafeteria lies beyond the entrance".

\subsection{Wayfinding - Procedures and tools}

In order to obtain a deeper insight in wayfinding mechanisms, we investigate individual procedures, components, aspects, and tools accompanied by the wayfinding process.

\subsubsection{Spatial memories - Cognitive maps vs. action-based representations}

In terms of human spatial memories a distinction is made between action-based representations (route knowledge) and the cognitive map or rather cognitive map representations (point and survey knowledge).

Route knowledge enables the wayfinder to follow a set of route segments. In general, these route segments are connections between landmarks. Having reached a landmark at the end of a route segment, the wayfinder adapts his direction of travel heading towards the next landmark (?) (for instance: if you are able to see the Empire State Building turn left and follow the avenue). Moving step by step from landmark to landmark the wayfinder finally arrives at the desired destination (path following) (?). At every step the wayfinder only knows the position of the next subdestination and is not able to establish an overview of the relative positions of the landmarks. He/she neither can point directly to the desired destination from the starting point of the route nor give information about a position of a subdestination unless it is the adjacent one. Hence, the wayfinder is not able to plan paths or routes, for instance short cuts, departing from the original route (??). Path following is accompanied by less effort as the wayfinder's tasks are restricted to the recognition of landmarks and the implementation of the instruction associated with the landmark. In many cases, human beings accomplish path following tasks without conscious reasoning, for instance when traveling on an absolute familiar route like the one from home to workplace (???). There is evidence that path following (responding to sensory-input and adapting the direction of motion) is achieved by using the caudate nucleus instead of parts in the hippocampus (??).

Point knowledge describes knowledge about the desired destination's location relative to the own position. There is no information about possible routes, route segments, subdestinations or landmarks located between the current position and the destination. Thus, the wayfinder has to look for paths or routes taking him/her at least closer to the destination (path searching). Obviously, routes leading (approximately) to the desired direction are appropriate (?).

Survey knowledge describes knowledge about multiple objects of the current environment, their relative positions to each other and possible connections between them. It can be compared to a highly developed cognitive map. The wayfinder has the possibility to evaluate his mental map and to plan an appropriate path to the desired destination (path planning) (?).

Both point and survey knowledge require the evaluation of cognitive map representations (objects' relations to the own location or other objects).

In most cases, the wayfinding process does not rely only on one single tool or strategy. In general, a successful solving of a wayfinding task makes use of multiple tools sequentially, simultaneously or both (?). 


\subsubsection{External aid or information}

To solve his/her wayfinding task, a wayfinder can look around for maps or signs possibly helping him/her. Given the case signs are available, the wayfinder can simply follow their instructions until he/she reaches a familiar place or the destination (?). The use or rather the memorization of maps leads to the establishment of internal spatial information, particularly to cognitive map representations (see Sec. 2.4.1) (?).

In certain situations, some people follow others and adopt their route choices assuming that the others are more familiar with the environment. Particularly in evacuation situations this behavior might be prevalent as the wayfinders are aware of the fact that they, most likely, pursue the same aim or the same spatial destination (find an exit). This behavior is called herding (??). As the strategy to follow others can remarkably influence the evacuation progress, we explicitly consider herding as a wayfinding strategy in evacuations.

\subsubsection{Generalized knowledge}

Human beings tend to classify new experiences (surroundings or situations) (??). They compare a new surrounding or situation with similar familiar ones which already have been well analyzed. The new experience can be categorized next to the comparable ones or rather in the same class. Once classified, the experience will be related to attributes, implications and expectations of the according class (??).

Clues about categories of experiences also influence the human ability and approach to solve wayfinding tasks (??). Although being faced with an environment never visited before, information about the type of the environment can be retrieved. This information does not incorporate the explicit spatial set-up of a building or an environment. Though, it gives clues about properties shared with other surroundings of the same type or category (??). We call this type of information generalized knowledge. Located in an arbitrary train station, for instance, people expect the possibility to enter trains via platforms. Leaving a subway train they expect the exit of the station somewhere upstairs. In offices and public buildings people know that they have to use circulation rooms to reach their destination successfully and efficiently.

Many conceivable search strategies are related to generalized knowledge. The expedient strategy to prefer circulation rooms in office buildings, for example, is based on generalized knowledge about the structure of office buildings. Even if the wayfinder has to select one of multiple proceeding ways that consist of the same room type, he/she possibly prefers one specific way. For example, it has been shown that people favor brighter and wider corridors which provide the greater field of view over other corridors (???).

\subsection{Computational models of wayfinding in the literature}

Computational models of wayfinding are widespread in the field of cognitive science, neuroscience, artificial intelligence, robotics, etc.. On the one hand, there are neural network/architecture approaches explicitly modeling mechanisms in the brain which are involved in the wayfinding process, incl. learning, forming, and retrieving cognitive maplike memories (??).

On the other hand, there are symbolic models modeling wayfinding and navigation in a more generalized way. They make use of rules and facts which are representative for neural procedures. Partly, the models, combined with sensory input functions, are implemented in robot systems enabling the robots to navigate autonomously. This includes the recognition of landmarks, destinations, and starting positions, determining the own location, and decision making (????). The reader is referred to ? for a comprehensive 
overview of symbolic models and neural networks.

Computational models are by this time elaborated regarding a plurality of aspects and principles of human wayfinding. Nevertheless, many of them are, considered as a whole, not well suited for an implementation within a simulation framework for pedestrian dynamics. This is caused by the fact that a majority of those models are created to serve different purposes (explain architecture and mechanisms of the hippocampus, robot navigation with a particular relatedness to sensory input functions, etc.).

Certainly various basic ideas of the mentioned models have to be considered when creating a wayfinding model for pedestrian dynamics simulations. In the field of evacuation dynamics implementations of human wayfinding processes are rare. However, there are already approaches to involve specific parts or aspects.

With the help of a queuing network ? investigated the impacts and consequences of discrete choices at the knots of the network depending on various types of behavioral pattern models. The behavioral patterns i.a. include a random choice, a path following (frequently used path), and a directional choice behavior. The frequently used path model makes agents ${ }^{4}$ tend to travel on a determined path. By a prescribed probability the agents lose the path and proceed by using random choices. If the directional choice model is applied, agents prefer graph segments (edges) leading them to a specific direction. To some extend the frequently used path and the directional choice model represent point and route knowledge.

? distinguishes between people that are familiar with a certain environment and those who are not or only to some extend. To model agents who have restricted information about their current environment, ? introduces spatial inaccuracies concerning metrics and direction. Furthermore, human wayfinding is represented by various models including route choices that depend on the direction to the destination (point knowledge), on the preference of routes that avoid turns, and on the movement of other people (herding). Additionally, landmarks are introduced serving as subdestinations (?).

? created a unified routing model by utilizing the models of ?. By unifying the models that represent persons with profound knowledge and persons who are unfamiliar with the environment his new approach grants the possibility to simulate pedestrians with several different knowledge degrees. In addition, the agents are using a mixture of appropriate tools and strategies to find their way, namely egocentric and allocentric memory ${ }^{5}$.

? present modeling approaches for search strategies (random search and nearest room heuristic) to find an exit without any knowledge about the spatial structure. The random search algorithm makes agents randomly select the next possibility to proceed. Agents proceed to the nearest located (measured by euclidean distance) crossing if the nearest room (or nearest door) heuristic is utilized. In both cases the agents avoid crossings leading to rooms they already have visited. The approaches are compared to a shortest path algorithm (in the paper called optimal solution).

? created an approach which combines a personalized spatial cognitive road network with implementations of selected routing strategies e.g. the use of the shortest path, most familiar path or the least crowded path.

Although several aspects of wayfinding are already taken into account in the previously mentioned models, there is still room for improvements. For instance, a unification of a knowledge representation which allows to model no, incomplete, inaccurate or wrong spatial knowledge with search strategies based on generalized knowledge is still missing.

\footnotetext{
${ }^{4}$ Simulated individuals following the rules of the model

${ }^{5}$ These types of memory can be compared to route and survey knowledge
} 


\section{New taxonomy of wayfinding tasks in evacuations}

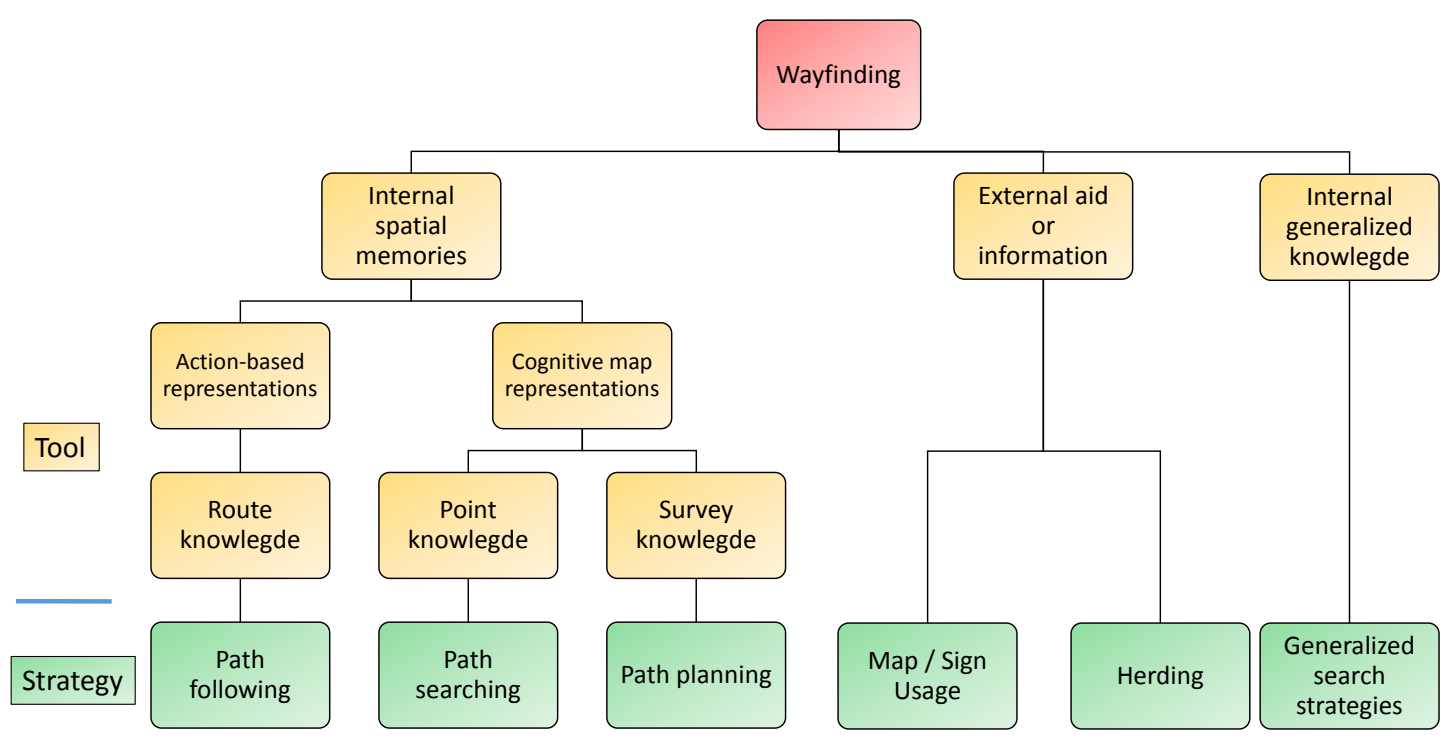

Figure 2. A wayfinding taxonomy showing tools and strategies which are used to solve wayfinding tasks in evacuation situations. The taxonomy is based on a taxonomy by ?.

In order to summarize the previously described scientific findings that are of particular interest to understand human wayfinding in evacuations, we propose a new wayfinding taxonomy. The taxonomy is an adapted and restructured version of a taxonomy proposed by ? (see Fig. 2). The purpose of the new taxonomy is to show the tools and strategies that are particularly used in evacuation situations. In principle, the taxonomy shows the above discussed separation between the individual wayfinding tools and their according strategies. In this context, a wayfinding strategy is the application of its according tool.

It has been distinguished between external clues recorded during the wayfinding process from sensory-input (see Sec. 2.4.2) ${ }^{6}$, internalized spatial memories from preceding visits including the subdivision into route, point and survey knowledge (see Sec. 2.4.1), and generalized experiences (see Sec. 2.4.3). With the help of route, point, or survey knowledge the strategies path following, path searching or path planning can be applied (see Sec. 2.4.1). In dependency on the type of available external clues maps or signs can be used or other people can be followed (see Sec. 2.4.2). Based on generalized experiences expedient search strategies e.g. use circulations (see Sec. 2.4.3) can be applied.

We assume that, in an evacuation situation, a person proceeds to or tries to find a specified destination, in most of the cases a specific exit or at least any exit. The destination could also be the position of a friend or a family member who has been separated from the wayfinder. However, undirected wayfinding behaviour, for example an purposeless pleasure walk, is very unlikely to take place in an emergency situation. Thus, in contrast to the taxonomy by ?, undirected wayfinding is excluded in the taxonomy proposed in this work.

\footnotetext{
${ }^{6}$ Not including the recognition of internalized objects (landmarks)
} 


\section{New wayfinding framework}

\subsection{Miscellaneous}

In this section, we propose a new human wayfinding model designed to be applied in pedestrian simulations. The approaches that are presented in the following incorporate the representation of internal explicit spatial knowledge (point, route and survey knowledge) (see Sec. 2.4.1) and approaches to model generalized knowledge (see Sec. 2.4.3). Furthermore, properties, items and mechanisms of the cognitive map are considered. Despite the fact that the model is designed for indoor wayfinding processes, it is extendable to outdoor wayfinding tasks, too. In the following enumeration we summarize the requirements the model has to cover in order to model the basic aspects and principles of explicit spatial and generalized knowledge.

The model is supposed to

- provide simulated agents with parts of information about the environment (instead of global knowledge),

- introduce spatial, particularly metric inaccuracies,

- be able to represent point, route and survey knowledge,

- take into account a cognitive map like structure including objects (landmarks) and their spatial relationships to each other,

- provide an appropriate approach to model generalized knowledge.

Underlying graph network
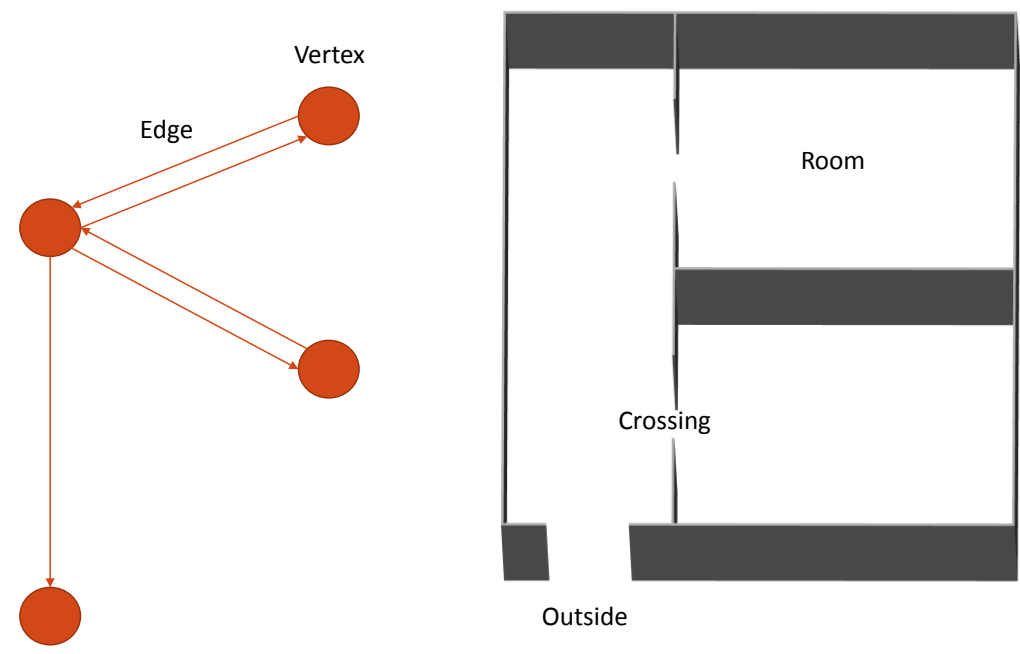

Figure 3. An example graph network implemented in JuPedSim. Rooms in the simulation scenario are represented by vertices of the graph network. Crossings are represented by edges connecting two vertices.

The model is implemented in the software framework Jülich Pedestrian Simulator (JuPedSim) (?). The spatial structure of a simulated environment is represented by a graph network $G=G(V, E)$ (see Fig. 3), where the rooms or rather convex areas are depicted by vertices $V:=B$ (a set of all rooms $B$ ) and crossings connecting the rooms are depicted by edges $E:=B \times B$. 


\section{Perceptional abilities}

Every time an agent enters a new room or enclosed area (described by a specific vertex), it can perceive all crossings (described by adjacent edges) leading to subsequent rooms or areas. Furthermore, it evaluates the crossings' positions relative to its own position. Therefor, the agent is able to evaluate accurately the euclidean distance between its and the crossings' locations.

Adjacent edges can be rated with a specific weight. The weights contain information about the adjacent room's type, whether the adjacent room has already been visited or if that room is a "good" choice to reach the final destination considering the knowledge degree of the agent. The agent has access to the weights of adjacent edges. It uses the crossing related to the edge $E_{\text {min }}$ possessing the lowest ("best") weight $w_{\min }$.

\section{Overview}

Adjacent crossings are weighted by three independent components of the model which consider an agent's individual (long term) spatial memories (the cognitive map), generalized knowledge and short term memories (see Fig. 4). These parts are described in detail in the following subsections. The weighting system evaluates the weights obtained by the three model parts and provides a final route choice decision. In Sec. 4.6 the weighting system is explained in detail.

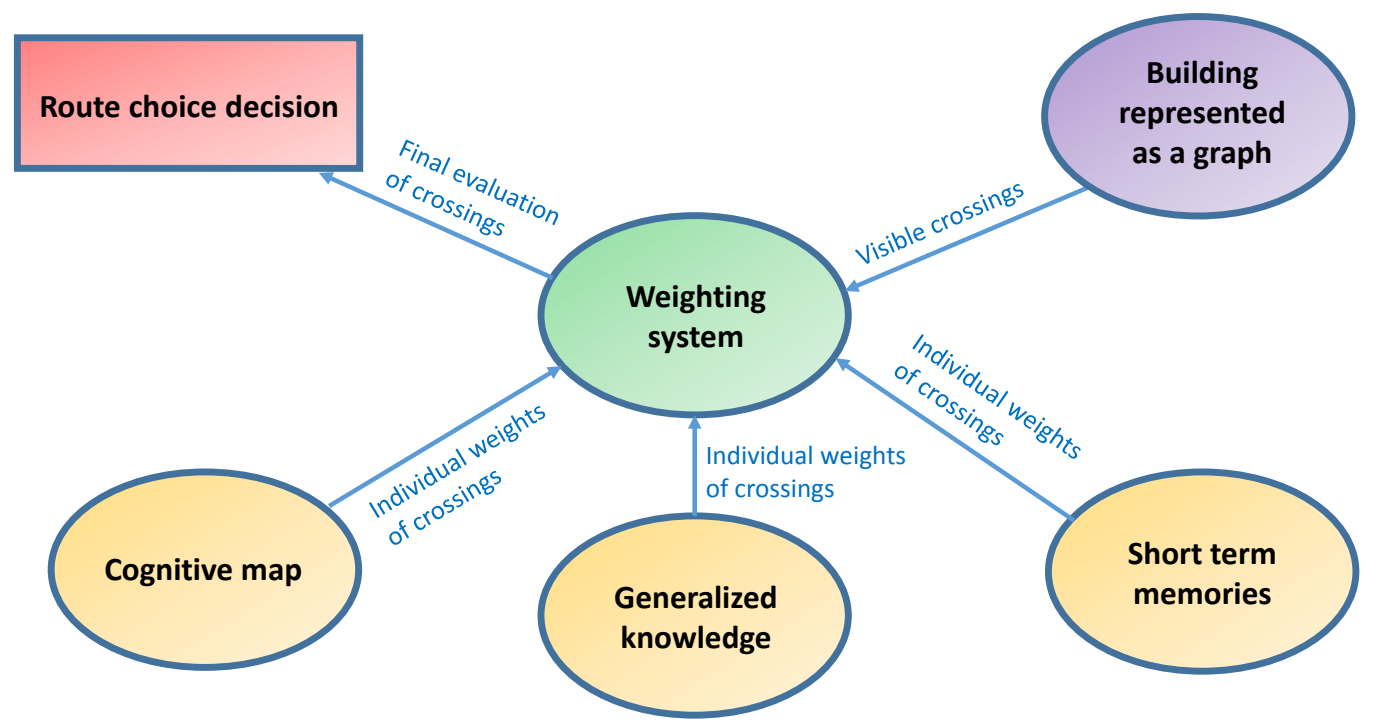

Figure 4. Overview of the three model components that influences an agent's route choice decision (orange). The weighting system (green) evaluates the weights given by the model parts and provides the final decision.

\subsection{Spatial learning concept of short term memories}

Every agent is capable to remember and re-recognize rooms he has already been in during the execution of the simulation. This ability is achieved by storing the vertices of the graph network that are related to the already visited rooms in an individual container for every single agent (??). Edges leading to a vertex which is part of this container are weighted differently (see Sec. 4.6). As mentioned above, this concept considers only spatial memories gathered after the start of the simulation. All individual containers are 
empty in advance. We call the knowledge about visited rooms short term memories to distinguish it from the cognitive map and generalized knowledge that, as the case may be, exist in advance before the start of the simulation.

\subsection{Modeling generalized knowledge}

To cover the findings mentioned in Sec. 2.4.3, we enable agents to recall generalized knowledge about their surroundings. The basic idea of this approach is that pedestrians favor certain types of rooms or enclosed areas over others in consideration of their generalized knowledge. In our implementation the agents differentiate between circulation rooms (corridors, entrances, stairs, lobbies, ramps, etc.) and rooms dedicated to a specific usage (except for the circulation purpose), for instance common rooms, store rooms or functional rooms (??). Circulation rooms are preferred as they principally lead efficiently to the destination. For this purpose, edges leading to circulation rooms are assigned with a weight that is lower ("better") in comparison to other edges (see Sec. 4.6).

The basic idea of considering generalized knowledge can be extended or transferred to scenarios taken place in other types of buildings, for example subway stations. Here, stairs leading upstairs might be favored.

\subsection{Modeling cognitive map representations}

As mentioned in Sec. 2.2, the human cognitive map only contains comprehensive and accurate information in very rare cases. By the modeling approach described in the following we provide the possibility to model incomplete, inaccurate or even wrong knowledge. To introduce spatial (especially metric) inaccuracies, the here introduced cognitive map approach does not contain the exact (zero-dimensional) positions of objects. Instead, positions of objects (landmarks) are described by ellipses (see Fig. 5). The ellipses represent the inaccuracy of a wayfinder's knowledge concerning both the angle and the distance to a landmark. The spatial knowledge degree can be manipulated by including or omitting landmarks or by changing size, shape or position of the ellipses. The exact position of the landmark does not necessarily have to be located within the ellipse. Hence, the modeled cognitive map might provide very inaccurate or even wrong clues about the relative positions of landmarks. In addition, the cognitive map holds information about known connections between landmarks (see Fig. 5).

Before the start of a simulation all agents are provided with an individual container that incorporates a specific amount of landmarks and connections.

\subsubsection{Evaluation of the cognitive map}

Fig. 6 shows an agent's possible cognitive map of a fictional office building. The cognitive map can be regarded as a mental representation of someone's survey knowledge as it is filled with a relatively high number of landmarks and connections. The spatial structure in the background is not part of the map. It is only shown for demonstration and explanation purposes.

Fig. 7 describes how the cognitive map is evaluated or rather how the next step or the next subdestination within the wayfinding process is determined. We regard the actual example case. Following the procedure shown in Fig. 7 the agent looks for the location of a main destination in the cognitive map. A "main destination" is a special landmark that represents a final destination, for example a known exit. If at least one could be found, the agent looks for a landmark in his nearer surrounding which is connected (possibly via other landmarks) to the destination(s). In the example case, the closest one (considering 


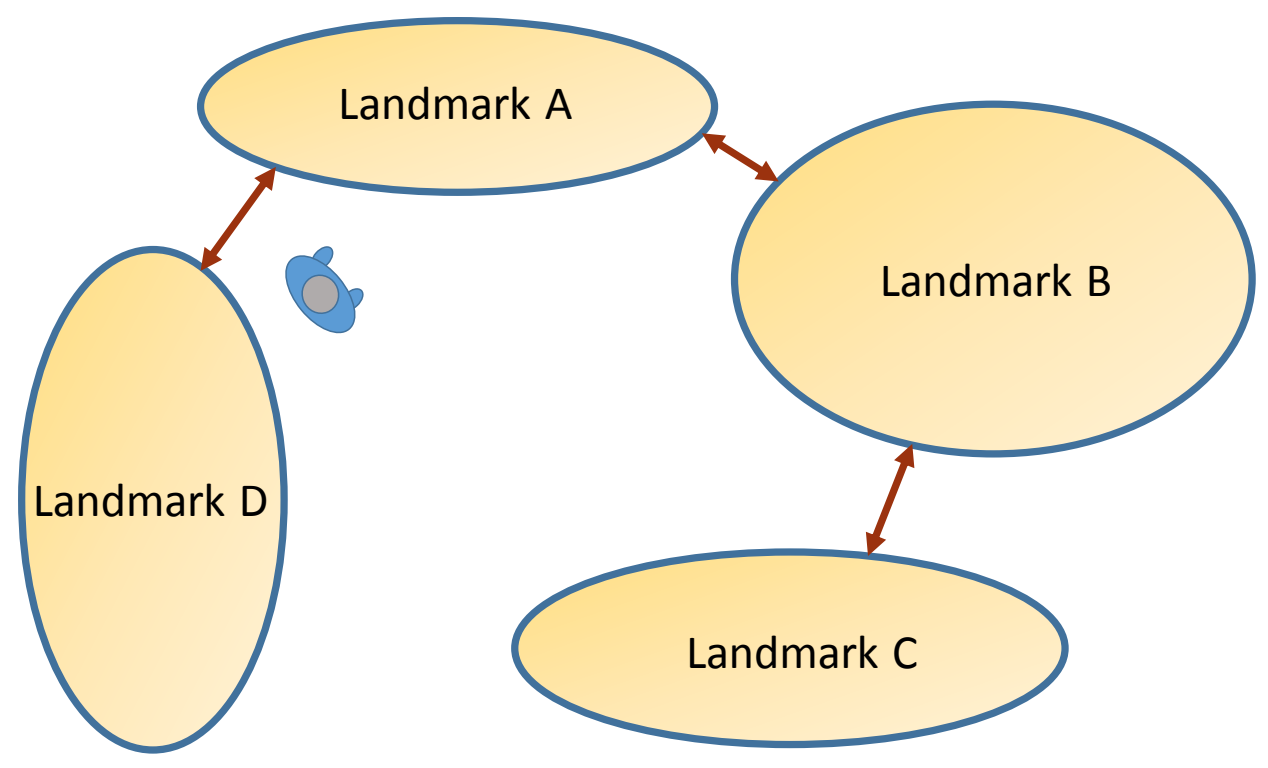

Figure 5. Representation of landmarks in a cognitive map. The ellipses depicts the inaccuracy of the knowledge about the landmarks' positions. Thin lines mark connections between landmarks.

the euclidean distance between the center of the landmark and the position of the agent) of all landmarks that are connected to the main destination is Landmark A (marked green).

There are several chains connecting Landmark A and the final destination. The shortest one is the chain A-B-C-F-Destination. Blue arrows are used to mark the chain in the figure. The lengths of the connection chains are calculated by accumulating the distances between two randomly determined points within two adjacent landmarks or rather their elliptical representations.

Since Landmark A is already in the agent's sight ${ }^{7}$, the next one in the connection chain to the destination, namely the landmark Landmark B (marked violet), will be treated as the next subdestination. The determination of the next subdestination is finished. Therefore, the path searching algorithm which is explained in the next subsection is initiated considering the landmark Landmark B as the next subdestination.

Every time the agent enters a new room, the above mentioned procedure is started. Having left the first room, the agent reaches the landmark Landmark B. Thus, (following the procedure again) the landmark Landmark $\mathrm{C}$ is identified to be the next subdestination. The procedure is repeated until the agent arrives at the main destination eventually.

Given the case a final destination does not exist in the map or it has been reached but still no exit is in sight, information from the cognitive map is not used (anymore). The agent has to rely on other information or rather strategies to make his next route choice decision.

\subsubsection{Path searching to landmark}

The above shown process describes how the next subdestination within the cognitive map is selected. However, it has not been explained how the agent moves to this destination or rather find its location, particularly if this destination is not located in the adjacent room. By the following we demonstrate the mechanisms executed if the agent is proceeding

\footnotetext{
${ }^{7}$ Landmarks which are in the same room are visible to the agent
} 


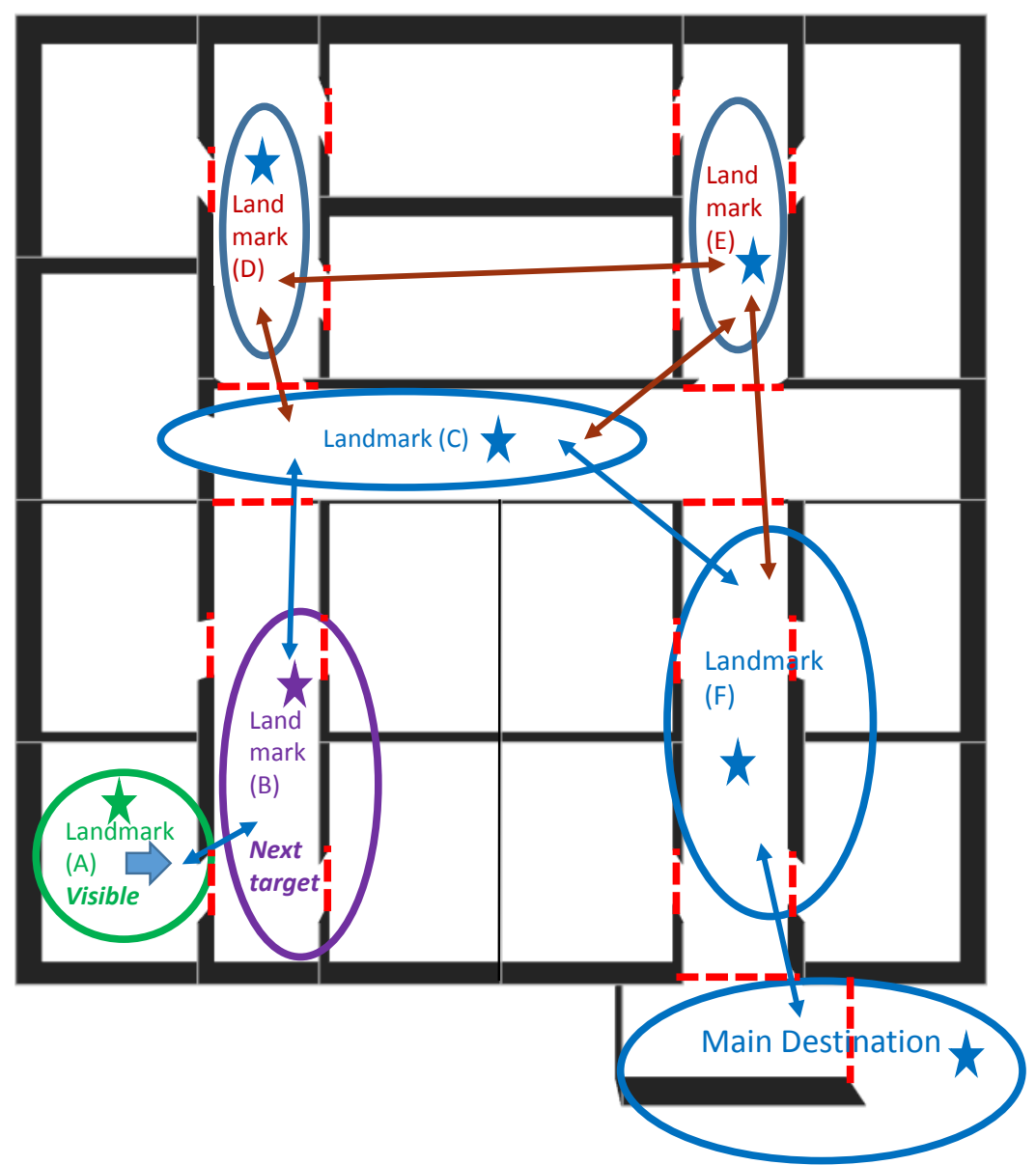

Figure 6. In the background: Spatial structure of a fictional office layout. In the foreground: A conceivable cognitive map of the structure in the background. The ellipses represent the inaccurate idea about the landmarks' positions within the map. The thin arrows depict connections between the landmarks. The current agent's location is marked by the blue bold drawn arrow. The stars mark in which rooms the real landmarks' positions are. In the context of the model it is not relevant where exactly the real positions of the landmarks are within the rooms as the agents can perceive any objects from any point within the room.

to the next subdestination. The same mechanisms are used if the cognitive map only provides the location of the main destination or very few (further away) landmarks (point knowledge).

In every room, the agent is supposed to select a doorway leading most efficiently closer to the next (sub-)destination or at least to an appropriate direction. However, it does not possess any knowledge about the spatial structure between its own and the landmark's position. Consequently, it can only make its decision based on knowledge about its immediate surrounding (in this case the actual room) and the (sub-)destination's position. Therefore, we introduce a procedure that enables the agent to make an educated guess in terms of which doorway shall be used to get most purposefully to the desired destination. For each agent this procedure is initiated every time it enters a new room (?).

First, the agent's actual room is regarded without exception. That means, walls and obstacles belonging to other rooms will be ignored in the context of this procedure (see Fig. 8). This is reasonable as the agent has no information about the spatial structure beyond its actual room except for the items in its cognitive map. The next step comprises 


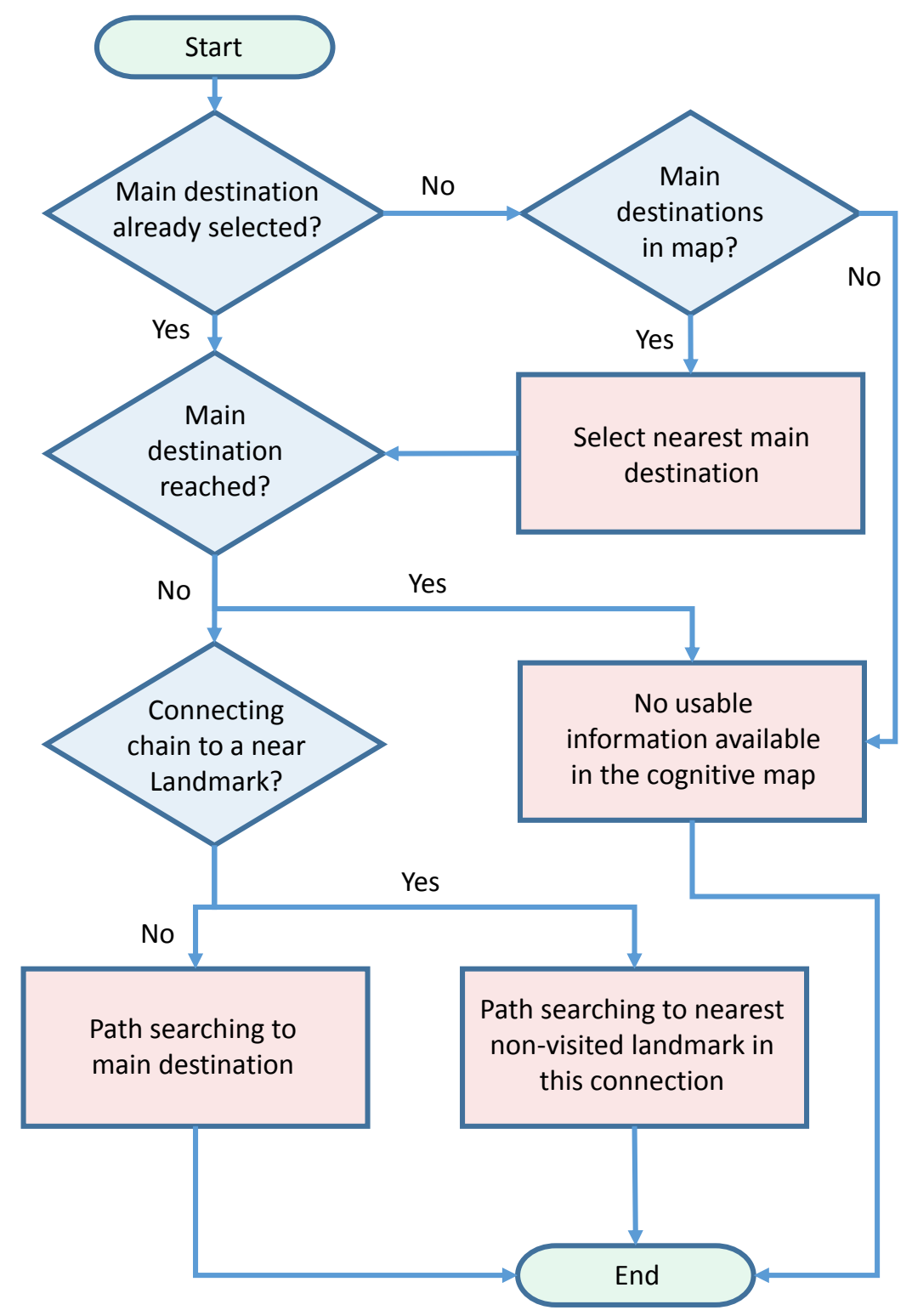

Figure 7. The figure shows the algorithm which is used to evaluate the items in the cognitive map and to determine a proper subdestination.

the calculation of all shortest paths (polygonal chains) connecting the crossings of the actual room and the ellipse representing the inaccurate idea of the (sub-)destination's location (see Fig. 8). The lengths of these polygonal chains are calculated under the constraint that the room must not be traversed by them but has to be bypassed (the polygonal chains must not overlap with the polygon that covers the area of the room) ${ }^{8}$.

With respect to their lengths the polygonal chains are ranked. In Fig. 8 the shortest one (related to rank no. 1) of the polygonal chains is shown dashed (green). The agent supposes that the door related to this path is the most proper possibility to reach the (sub-)destination. All edges corresponding to the adjacent doorways are weighted in consideration of their rank (see Sec. 4.6). If no other factors influence the route choice decision, the agent chooses the doorway related to rank no. 1. Although this procedure

${ }^{8}$ The calculation is achieved with the help of the C++ library VisiLibity (?). 
is not expedient in every case (could lead into dead ends for instance), it is reasonable enough to get closer to the (sub-)destination taking into account the lack of comprehensive information about the spatial environment.

It is conceivable to consider the paths starting from an agent and proceeding via a crossing to the ellipse instead of considering solely the paths starting from the crossings. However, in particular cases, this procedure would move the agents to use crossings in the opposite direction of the target ellipse. For instance, this might happen when an agent is located very close to a crossing. Thus, the here described path searching procedure is the better choice to represent the implementation of an educated guess of where the next (sub-)destination is positioned.
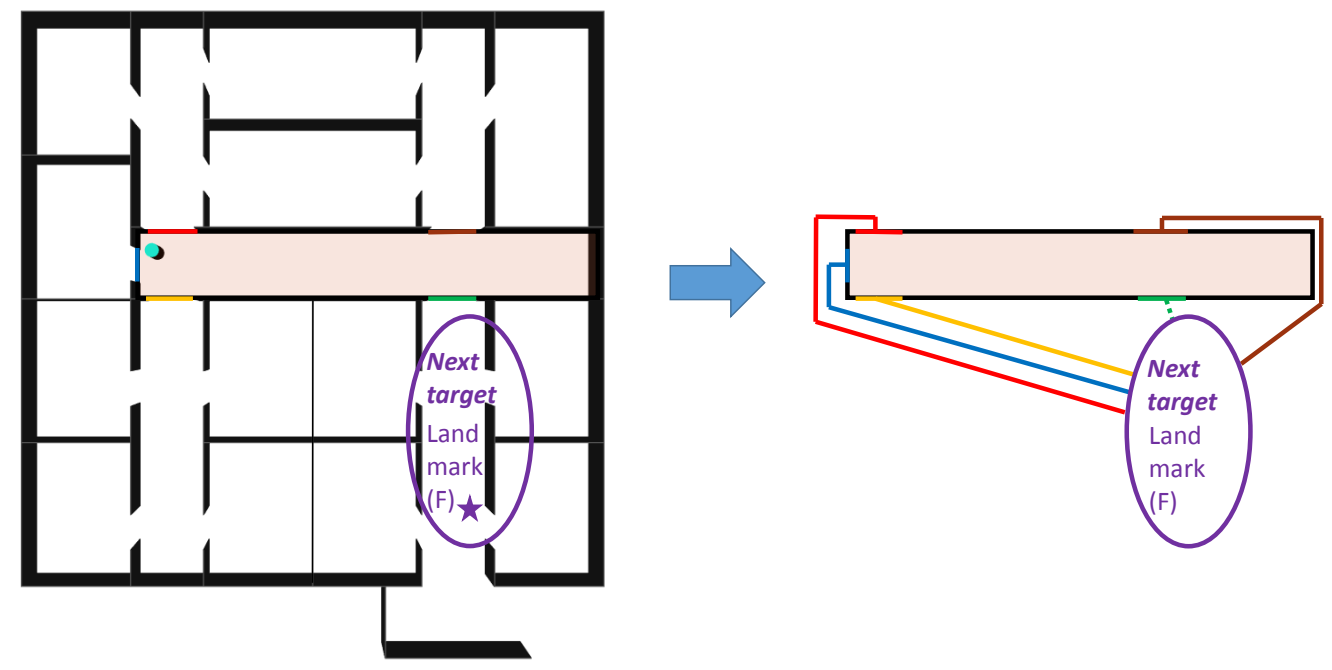

Figure 8. On the left: Possible cognitive map of an agent with only a single clue about its spatial environment (point knowledge). On the right: Actual room and target ellipse regarded without exception. Shortest paths (polygonal chains) from the doorways of the room to the ellipse are displayed. The agent assumes the path that is shown dashed to be the best choice to get closer to the landmark.

It is possible that the agent is already located inside the ellipse but cannot see the according landmark (real position of the landmark is not in the actual room). For example, this can happen if the real position of the landmark is apart from the position of the ellipse. In this case, the information in the cognitive map won't be used. The agent will utilize other strategies to proceed, e.g. explore the adjacent rooms by heading to the nearest located door or by choosing randomly (see Sec. 4.7).

\subsection{Modeling action-based representations (route knowledge)}

As described in the previous section, route knowledge relies on action-based representations. That means, following a route or using route knowledge does not require cognitive reasoning such as evaluating relations of multiple spatial objects (own position and landmarks). In case of recognizing a landmark, a wayfinder who relies on route knowledge is provided with a memory indicating the next route segment leading to the next landmark (subdestination). Although route knowledge is not belonging to the group of cognitive map representations, we propose mechanisms representing route knowledge based on approaches described in the above sections. 


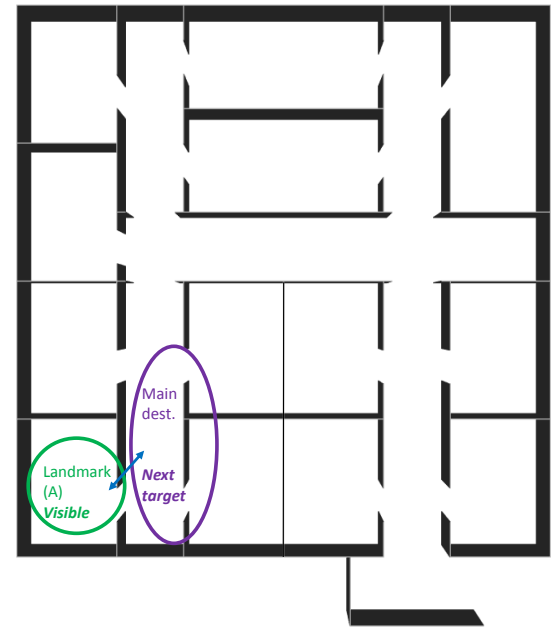

(a)

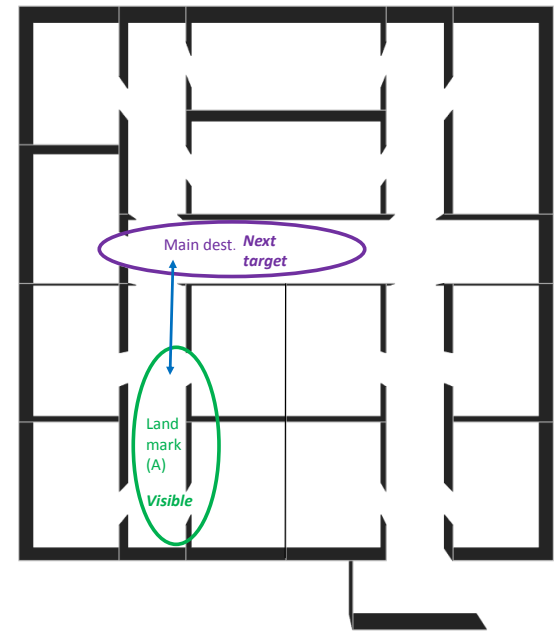

(b)

Figure 9. a)/ b) Possible current states of route knowledge. Route knowledge always incorporates two connected landmarks.

Fig. 9 depicts the mental representation of an agent's route knowledge. The representations always include two landmarks and the clue that a connection between them exists. The first landmark is in the visibility range of the agent (in the same room). The second landmark which also serves as the next subdestination is not visible at first. When the second landmark becomes visible during the agent's movement, new memory is retrieved. That means, the first landmark is erased from the current memory, second landmark becomes first and a new (second) landmark is retrieved. Every time the agent reaches a new room, the path searching procedure that has been described in Sec. 4.4.2 is initiated considering the second landmark as the new (intermediate) main destination. The procedure is repeated until the agent arrives at the main destination (of the whole route).

\subsection{The weighting system}

Edges of crossings of the room in which the agent is actually located are weighted. Short term memories, the cognitive map and generalized knowledge contribute to an alteration of a specific edge's weight. The calculation of a visible edge's weight $w$ is as follows:

$$
w=f_{\text {expl }} \cdot f_{\text {gen }} \cdot f_{\text {shortmem }} \cdot f_{\text {final }}
$$

$f_{\text {expl }}$ describes the factor that considers the cognitive map. $f_{\text {expl }}$ is calculated by

$$
f_{\text {expl }}=1-0.5^{i}
$$

$i=1,2,3, \ldots$ describes the index of the currently regarded edge in the ranked list of crossings in the context of the path searching algorithm (see Sec. 4.4.2). If no explicit spatial information is available, $f_{\text {expl }}$ equals 1.0.

The factor $f_{\text {gen }}$ takes into account the preference of certain types of rooms based on generalized knowledge. Weights of edges leading to circulation rooms are provided with the factor $f_{\text {gen }}=0.25$, other rooms are provided with $f_{\text {gen }}=1.0$.

In addition, edges are weighted considering the factor $f_{\text {shortmem }}=2.0^{k}$. Here $k=$ $0,1,2, \ldots$ describes how many times the adjacent room to which the edge leads has been 
visited.

Weights of edges leading to the outside obtain the factor $f_{\text {final }}=0.1$. Other edges are weighted with the factor $f_{\text {final }}=1.0$.

The shown factor setting comes along with the following consequences for the route choice of an agent:

- The agent prefers rooms which have not been visited.

- Rooms which only have been explored once are favored over rooms that have been visited twice (analog for higher numbers of visits).

- Circulation rooms are favored although that means the agent has to deviate remarkably from the beeline to the supposed position of his next destination.

- Circulation rooms are favored even if that means the agent has to turn around and go back to an already known circulation room.

- If a circulation room has been visited twice, it is no longer guaranteed that this room is favored over an unknown room of any type.

- If the product of $f_{\text {gen }} \cdot f_{\text {shortmem }}$ is equal for multiple edges, the most desirable edge will be determined by the factor $f_{\text {expl }}$.

- Final exits or rather crossings leading to the outside are favored in every case.

If two or more edges possess the same weight, one of them is randomly chosen or alternatively the nearest located one is regarded as the best choice (see Sec. 4.7).

\subsection{Search st}

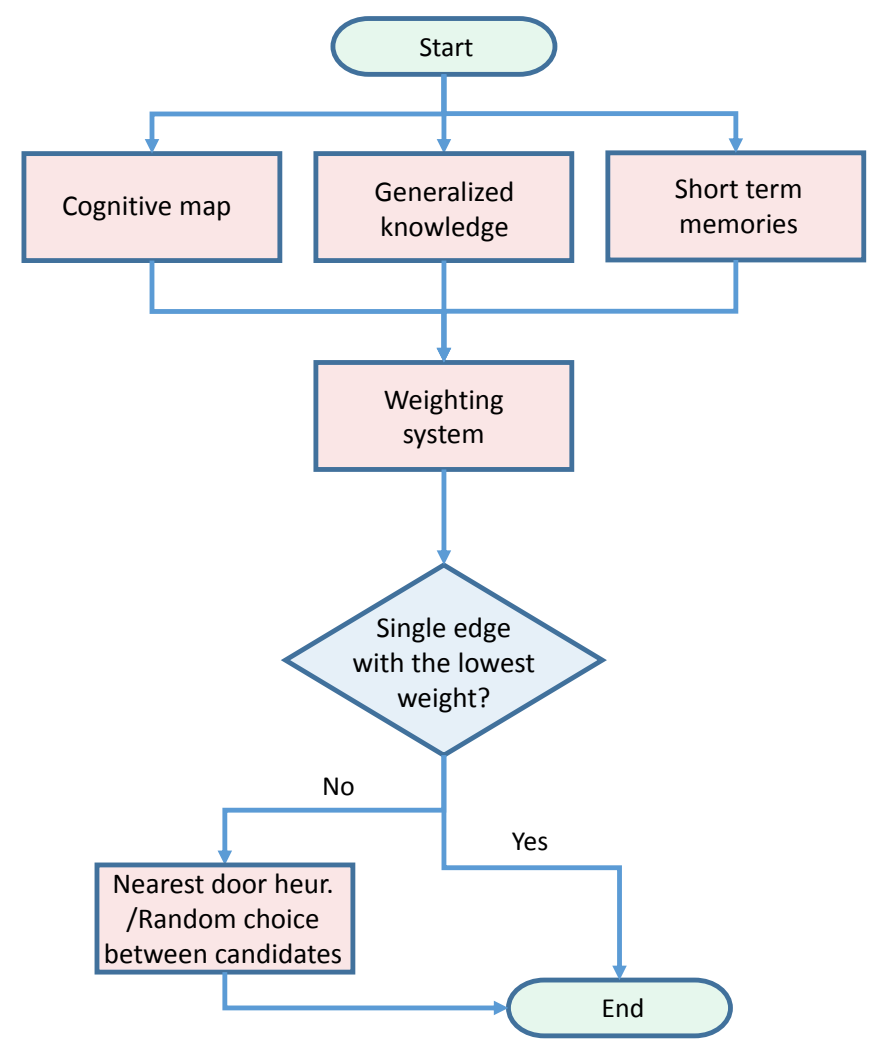

Figure 10. Factors that affect the weighting of a crossing. In case multiple crossings are weighted equally by these factors, random choices or the nearest door heuristic can be applied in order to decide between them.

Search strategies will be applied by agents if their cognitive map or action-based representations do not provide any expedient information. Generalized knowledge might 
be helpful in many of those situations. However, there still exist cases in which agents have to choose between edges that possess the same weight. This is the case if multiple crossings are available that lead to the same type of room. In these cases, we apply the nearest (local) door heuristic and random choices (??). The implementation of other strategies which might reproduce explorative behaviour more realistically is conceivable as well. In particular, ? found out that people tend to move straight forward unless the deviation from the current direction of movement provides a greater field of visibility or rather a broader view. This strategy might interfere with the favoring of circulation rooms in some cases. ?? showed that people prefer to use brighter and wider corridors. However, in emergency situations human beings might avoid long corridors in which no exit is in sight and rather turn to search for an exit which is in the nearer surrounding. For now, the here presented model is not capable to apply these exploration strategies as the agents' perceptional abilities are restricted to the recognition of adjacent doorways and the euclidean distances to them. Individual isovists or rather visible areas are not considered.

\subsection{Examples of the collaboration of the model's parts}

In this subsection, we investigate an agent's movement pattern under consideration of the wayfinding model that has been introduced previously. For this purpose, we make use of the fictional building's floor depicted in Fig. 11. A single agent is located in the left lower corner of the floor and tries to reach the exit which is located underneath the corridor in the right lower corner.

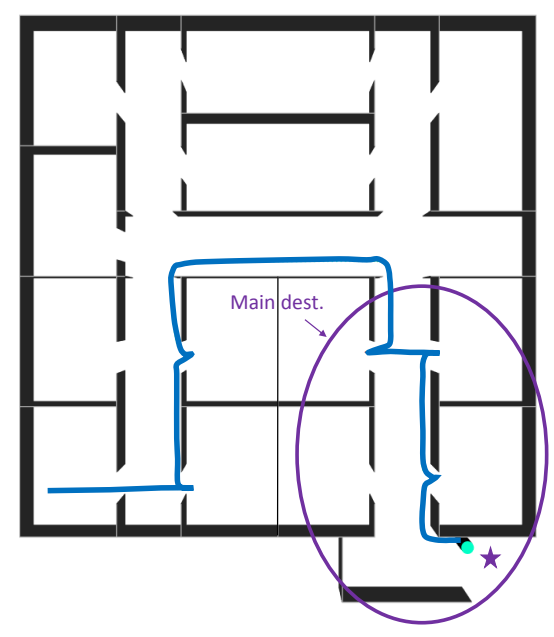

(a)

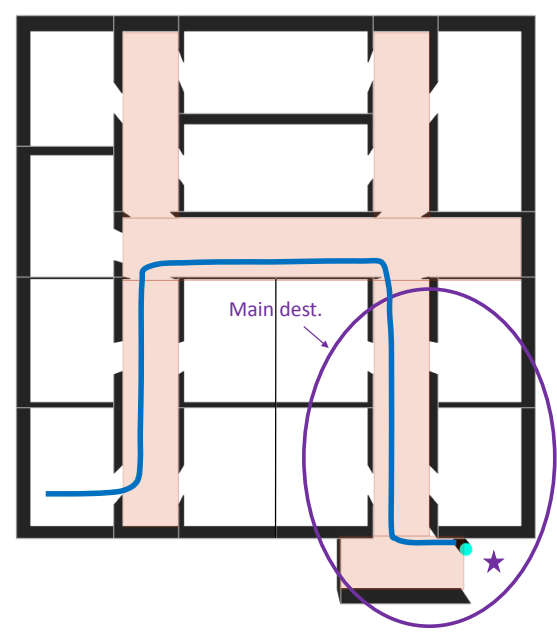

(b)

Figure 11. Trajectories of an agent starting in the left lower corner and trying to find the exit. a) The agent possesses a single clue in its cognitive map. b) The agent possesses a single clue in its cognitive map and knows about the implications of circulation rooms.

Both example scenarios comprise the simulation of an agent provided with an inaccurate idea about the exit's location (point knowledge). Its cognitive map is filled with one single item. In each of Figs. 11(a) and 11(b) the idea about the landmark's location is depicted by the ellipse. In these examples, the nearest door heuristic is applied if the edges of multiple crossings possess the same weight.

In example a) the agent relies on his point knowledge without exception. The agent uses doors taking him purposefully (see path searching procedure above) closer to the destination (see Fig. 11(a)). 
Having started its journey, the agent crosses the first corridor (lower left corner) heading to the opposite doorway as this crossing is obviously the best choice to come closer to the exit area. However, here, the agent recognizes that it is located in a dead end. In consequence, it turns around trying to reach the exit area by moving through the crossing to the adjacent room. Eventually, it arrives at the corridor located in the middle of the building which enables it to travel to the right lower region of the building.

In the following corridor on the right lower side the agent's position is inside the ellipse which represents the landmark. Its cognitive map indicates, that it should be already close to the landmark. However, it still has no visual contact to it. Inside the ellipse the path searching procedure will not be initiated anymore and the agent proceeds by exploring the environment. It heads towards the nearest doorway.

Example b) comprises the combination of generalized and cognitive map knowledge. To highlight the effects of this combination, the agent is simultaneously provided with the ability to distinguish between common rooms and circulation rooms and with a directional sense of the exit's location. For this purpose, the color coded rooms in Fig. 11(b) are indicated as circulation rooms. The agent assumes the strategy to move to or to stay on a circulation room to be more expedient as to keep the direction to the destination (see Sec. 4.6). In consequence, having left the starting room, the agent proceeds to the corridor in the middle of the building as it is the only adjacent corridor. In the middle corridor the agent has to choose between three corridors. Obviously, the corridor in the right lower corner is the best possibility to come closer to the exit area. Within the exit area the agent again prefers the only proceeding corridor that takes it eventually to the outside.

In this example scenario the agent is moving to the destination without making any detours. Hence, in this example case the search strategy (go to and stay on circulation rooms) and a vague idea about the location of the destination are sufficient to find a short (in this case the shortest) route to the outside.

\section{Calibration}

In this section, we show that, after a proper calibration, the above discussed modeling approaches can approximately reproduce the route distribution of a field study in which spatial knowledge played a major role.

\subsection{The field study}

\subsubsection{Method}

For calibration purposes we conducted a field study in a building of the Research Centre Jülich. The study took part on the second floor of the building. The spatial structure of this floor is depicted in Fig. 12. The floor is used (as well as the other parts of the building) for office purposes. The main goal of the study beside the model calibration was to investigate the route choices of participants with respect to their spatial knowledge degree about the building. During the experimental runs we covered the emergency exit signs to exclude the possibility that signage has an influence on the route choices. We only kept the signage which was directly installed on exit doors.

\section{Participants}

47 persons participated in the study. 23 of them were female, 24 male. The ages of the participants were between ca. 20 and ca. 70 years. The average age was approximately 
35 years. All participants were healthy and not physically impaired. They had no visual impairment or wear glasses to correct a visual impairment. The group of subjects mainly comprised either students or employees from the Research Center Jülich. The participants signed an informed consent agreeing to the record of their data. Data has been recorded anonymously and is used for scientific purposes only.

The subjects participated individually (sequentially). Beforehand, they were asked to fill a very brief questionnaire. Besides gender and age, in this context, the subjects were supposed to rate (from 1 to 10 ) their knowledge degree or rather their familiarity with the building. The results of the self-rating are shown in Tab. 1. In particular, the table shows the number of subjects that ranked themselves into a certain familiarity level.

Table 1. Results of the questionnaire: Self-rated familiarity of the participants.

\begin{tabular}{|c|c|c|c|c|c|c|c|c|c|c|c|}
\hline Knowledge degree & 1 & 2 & 3 & 4 & 5 & 6 & 7 & 8 & 9 & 10 & Total \\
\hline Number subjects & 5 & 3 & 3 & 4 & 2 & 1 & 7 & 13 & 5 & 4 & 47 \\
\hline
\end{tabular}

\section{Task}

Sequentially, the participants were led to the starting point via Balcony E (see Fig. 12). In this way, they could not see any part inside the building before the start of the study. At the starting point, the study's task has been explained to the subjects by the experimenter. The task incorporated the following points:

(1) The attendees were supposed to assume that they were in a situation of a hazard (for example a fire) and an announcement to leave the building has been given.

(2) It was not allowed to use Balcony $\mathrm{E}$ or $\mathrm{F}$ for the evacuations. The subjects were told that these exits were blocked for some reason (collapse, fire, smoke, etc.).

(3) The subjects were told not to run but walk normally.

For documentation purposes the attendees were asked to wear a headband on which a video camera has been installed.

Fig. 12 shows the routes the subjects could have used to fulfill their task, namely to evacuate from the building (considering the conditions mentioned above). Moreover, we assumed that nobody will try to evacuate by moving into a store room or an office due to generalized knowledge about office layouts. So we determined the possible routes by considering the corridors, foyers and stairs without exception. Routes A and B lead immediately to the staircases (Stairs D or G). Route C leads to Balcony J which can be seen on the right side of the floor plan. The crossing to Balcony $\mathrm{J}$ is not visible from the starting point. In fact, it only can be seen from a close position in front of it (see Fig. 13(a)). Each of the routes D, E, F, G, H, I, J, K lead either to the corridor at the upper or at the lower side of the ground plan. Within these corridors, one can go either immediately to one of the balconies B or I (Route I or Route D) or immediately to the staircases $\mathrm{C}$ or $\mathrm{H}$ (Route $\mathrm{H}$ or Route $\mathrm{E}$ ). Alternatively, it is possible to explore the corridors first and proceed then to a balcony or staircase (routes F, G, J, K). Both mentioned corridors possess pillars and furniture that hide the view of the whole corridor from several positions so that explorations into the corridors had been expected.

In connection to the upper corridor there is a possibility to proceed to another building (A). During the study the crossing to the neighbor building has been blocked. Subjects who wanted to pass here had to turn around and look for other options to evacuate. Route L describes the path from the starting point to the left corridor on the floor plan. It was not explicitly forbidden to use the left corridor. However, Balcony $\mathrm{F}$ has been blocked. Thus, participants who went here had to go back to the foyer where the starting 


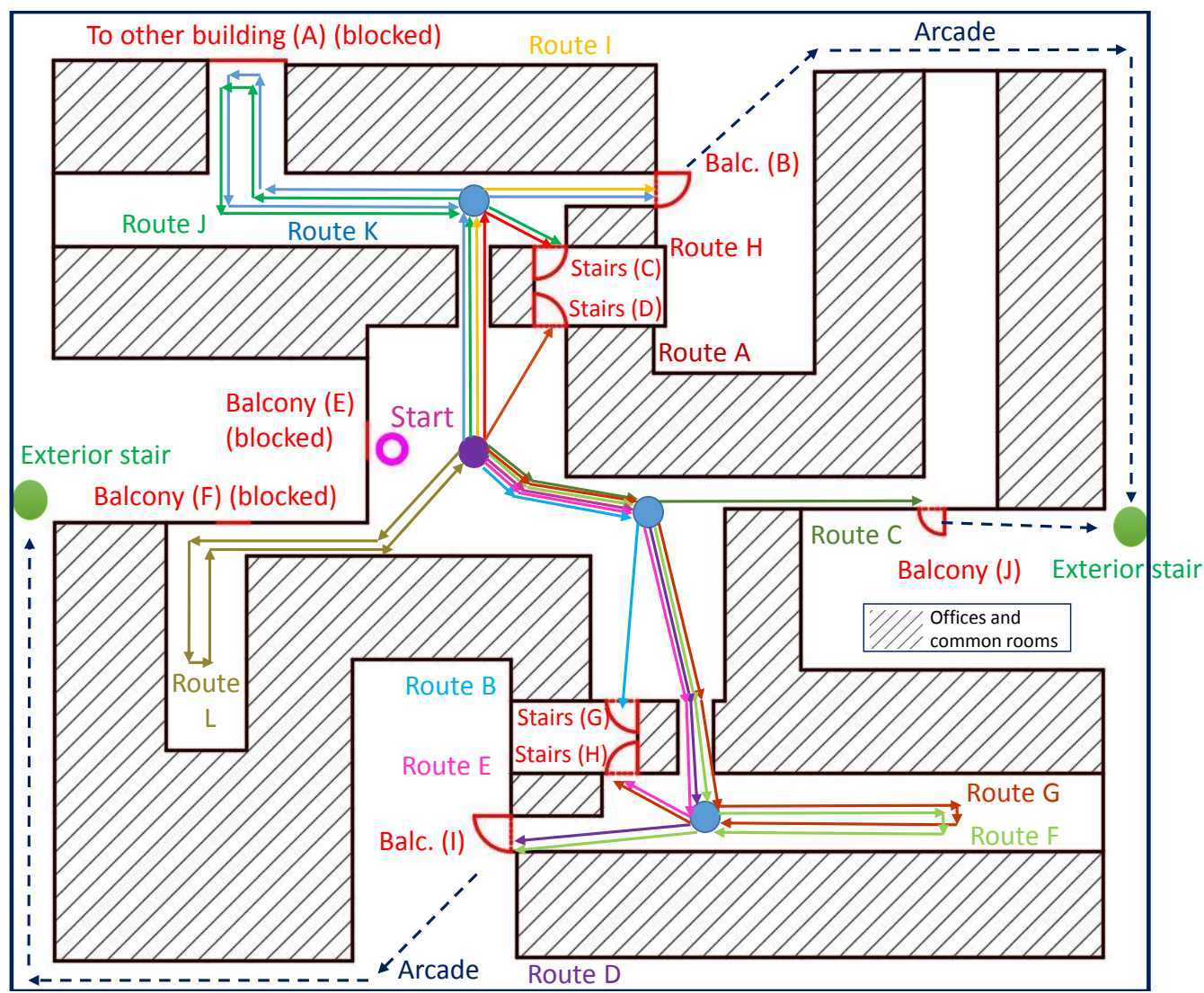

Figure 12. In the background: The floor plan of the area which was used for the field study. In the foreground: All possible routes the subjects could use.

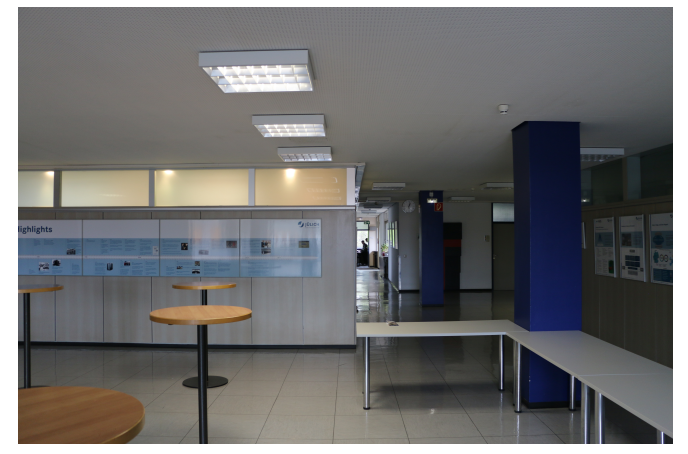

(a)

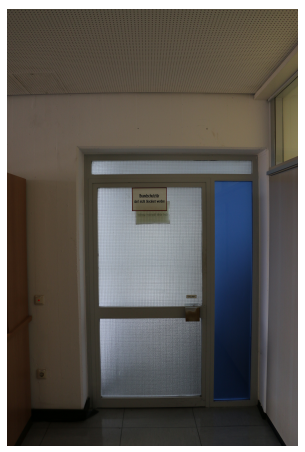

(b)

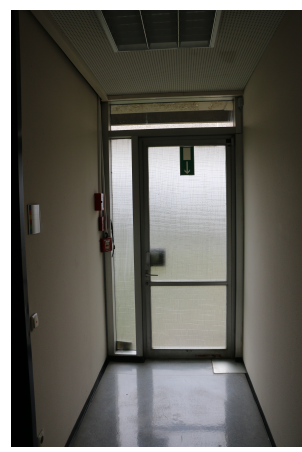

(c)

Figure 13. a) View from the starting point towards the corridor on the right side of the floor plan. b) Crossing that leads to Stair D. c) Crossing that leads to Balcony B.

point was located. Back in the foyer, they had to start their journey again and decide for one of the other mentioned routes. As only one subject took Route L, we do not consider which route has been taken after the subject came back to the starting point. In consequence, all routes that starts with a journey to the left corridor are regarded as Route L.

Each of the doors that either lead to one of the balconies or one of the staircases consists of non-transparent but translucent glazing (see Fig. 13(c)). The door at Balcony $\mathrm{J}$ is an exception as it consists of transparent glazing. Doorways that lead to staircases are not furnished with signs (see Fig. 13(b)). Doorways to the outside are provided with green exit signs (see Fig. 13(c)) which were not covered (in contrast to the rest of the signage 
in the building).

Via one of the staircases it is possible to move downstairs and proceed via a corridor to find a final exit on the ground level eventually. Alternatively, participants could use one of the exterior staircases to reach the ground level. These staircases are directly accessible via balconies $\mathrm{J}$ and E/F. From balconies B and I they can be approached by following the exterior arcade that leads around the second floor of the building.

For reasons of simplification, in this paper, we regard the route choices on the second floor without exception.

\subsubsection{Results}

Tab. 2 shows the route usages that resulted from the field study. Particularly, the table mentions how the subjects who took a specific route rated their own knowledge degree. For example: 3 participants who rated their knowledge degree with the value 8 took Route C (see row 5 / column 9 of Tab. 2).

It is clearly visible that the route usage of participants that possessed a minor knowledge degree $(1-5)$ comprises the use of more different routes ( 8 routes) in comparison to the route usage of the familiar participants (6-10) (3 routes). One participant of those who classified their knowledge degree in the interval from 1 to 5 took Route A, five took Route B, another four used Route C, one Route G, one Route J, two Route K and another one Route L.

Table 2. Distribution of routes that were chosen from the study's participants in consideration of their self-rated knowledge degree. The average knowledge degree is about ca. 6.1.

\begin{tabular}{|c|c|c|c|c|c|c|c|c|c|c|c|}
\hline Route / Knowledge degree & 1 & 2 & 3 & 4 & 5 & 6 & 7 & 8 & 9 & 10 & Total \\
\hline \hline Total number & 5 & 3 & 3 & 4 & 2 & 1 & 7 & 13 & 5 & 4 & 47 \\
\hline \hline Route A & - & - & - & - & 1 & - & 4 & 7 & 1 & - & 13 \\
\hline Route B & - & 2 & 1 & 2 & - & 1 & 1 & 3 & 1 & - & 11 \\
\hline Route C & 2 & 1 & - & 1 & - & - & 2 & 3 & 3 & 4 & 16 \\
\hline Route D & 1 & - & 1 & - & - & - & - & - & - & - & 2 \\
\hline Route E & - & - & - & - & - & - & - & - & - & - & 0 \\
\hline Route F & - & - & - & - & - & - & - & - & - & - & 0 \\
\hline Route G & - & - & - & - & 1 & - & - & - & - & - & 1 \\
\hline Route H & - & - & - & - & - & - & - & - & - & - & 0 \\
\hline Route I & - & - & - & - & - & - & - & - & - & - & 0 \\
\hline Route J & - & - & 1 & - & - & - & - & - & - & - & 1 \\
\hline Route K & 1 & - & - & 1 & - & - & - & - & - & - & 2 \\
\hline Route L & 1 & - & - & - & - & - & - & - & - & - & 1 \\
\hline
\end{tabular}

Exits to Balcony I or Balcony B were visible from within the upper and lower corridor. Nevertheless, here, 3 participants decided to go left and were forced to turn around due to the dead end at the end of the corridor. It is conceivable that these participants made their route choice decision and headed left before the exits were visible. Another possible explanation comprises the supposition that they saw the exit doors but could not identify them as doorways to the outside.

Even though the route usage of unfamiliar subjects varies, the results listed in Tab. 2 show a slight preference for Route B ( 5 subjects) and Route C (4 subjects). On the one hand, these subjects simply might have known these paths although their overall knowledge about the building has been rated to be minor. On the other hand, the preference might have turned out because some participants were attracted by the brightness of 
the corridor next to Balcony $\mathrm{J}$ or by the wideness of the foyer that is contiguous to the starting area.

Although the usage of Route L was possible, only one subjects decided to take this route. This might be caused by the fact that the experimenters told the participants that Balcony $\mathrm{E}$ and $\mathrm{F}$ are not usable for the evacuation. Consequently, the participants mainly preferred to head to directions moving away from the blocked balconies.

Participants who were familiar with the building took one of the routes A, B or C without exception. This clearly shows that spatial knowledge has a remarkable influence on the participants' route choice decisions. The high proportional usage of Route A and B (via stairs) can be explained based on the fact that the staircases are commonly used to enter and leave the second floor either from or to workplace, to administrative offices or meeting rooms. In fact, a number of participants stated after they left the building that they evacuated via the path they commonly use for any purposes and know for sure. They might have known about other routes as well but felt more comfortable using their daily path. Route $\mathrm{C}$ which is the shortest path to get to the outside (and to the ground level) has mostly been taken by subjects with prior knowledge about the building. All four subjects who claimed their knowledge to be comprehensive (value 10) used Route $\mathrm{C}$ without exception. This fact suggests that people who are very familiar with their spatial surroundings and are confident to evaluate and compare different routes in context of their length and travel duration prefer to use the shortest path.

\subsection{Calibration by comparing route distributions from field study and simulations}

\section{Preliminary considerations}

In this section, we show that the modeling approaches introduced and discussed in this paper are able to reproduce adequately the route distribution that resulted from the field study.

The calibration has been done for the cognitive map modeling approach. Due to the limited space in this paper the representation of route knowledge (see Sec. 9) is not considered or discussed explicitly within the calibration.

Each of the participants moved only on circulation rooms or rather did not walk into offices even though their doors were partially opened. Thus, in the context of the model calibration, agents are provided with generalized knowledge so that all of them prefer to use circulation rooms. If the weights of two or more accessible edges are identical, the agents randomly choose between the possibilities.

The route distribution of the calibrated model is compared to the distribution of the field study. In addition, route usages that resulted when using a shortest path algorithm, the nearest door heuristic and randomly made decisions are also regarded for comparison purposes. Regardless of the used routing model the decisions are always restricted to circulation rooms.

In the context of this calibration, the most decisive question is how the subjects or rather their knowledge degrees can be represented by simulated agents or rather their cognitive maps. To address this question, we start from the following premises:

- Agents that represent subjects who are absolutely familiar with the building are supposed to be provided with a comprehensive cognitive map that includes all relevant landmarks and possible connections between them. The position of these landmarks shall be accurate so that the agents are able to evaluate and favor routes that are shorter.

- We assume that participants who rated their knowledge degree with the value 1 are 
first time visitors. Accordingly, in the simulation they are provided with an empty cognitive map.

- Agents that represent subjects who claimed to have some but not comprehensive information shall obtain partially filled cognitive maps or rather cognitive maps in which specific landmarks and their connections are omitted.

- For the reason of simplification, we do not vary position, shape or size, but only the number of landmarks in the maps. Additionally, all connections between existing landmarks are kept.

\section{The calibration}

Fig. 14 describes the cognitive map of an agent with comprehensive spatial knowledge. Main destinations A and B represent the entrances of the building on the ground level or rather the exterior staircases that are located next to the entrances. Main destination $\mathrm{C}$ depicts the entrance of the neighboring building. As this entrance is far away from the other landmarks and the area of the study, the entrance's position is not shown explicitly in the figure. The remaining landmarks depict the positions of corridors and staircases that are relevant and useful for the evacuation task.

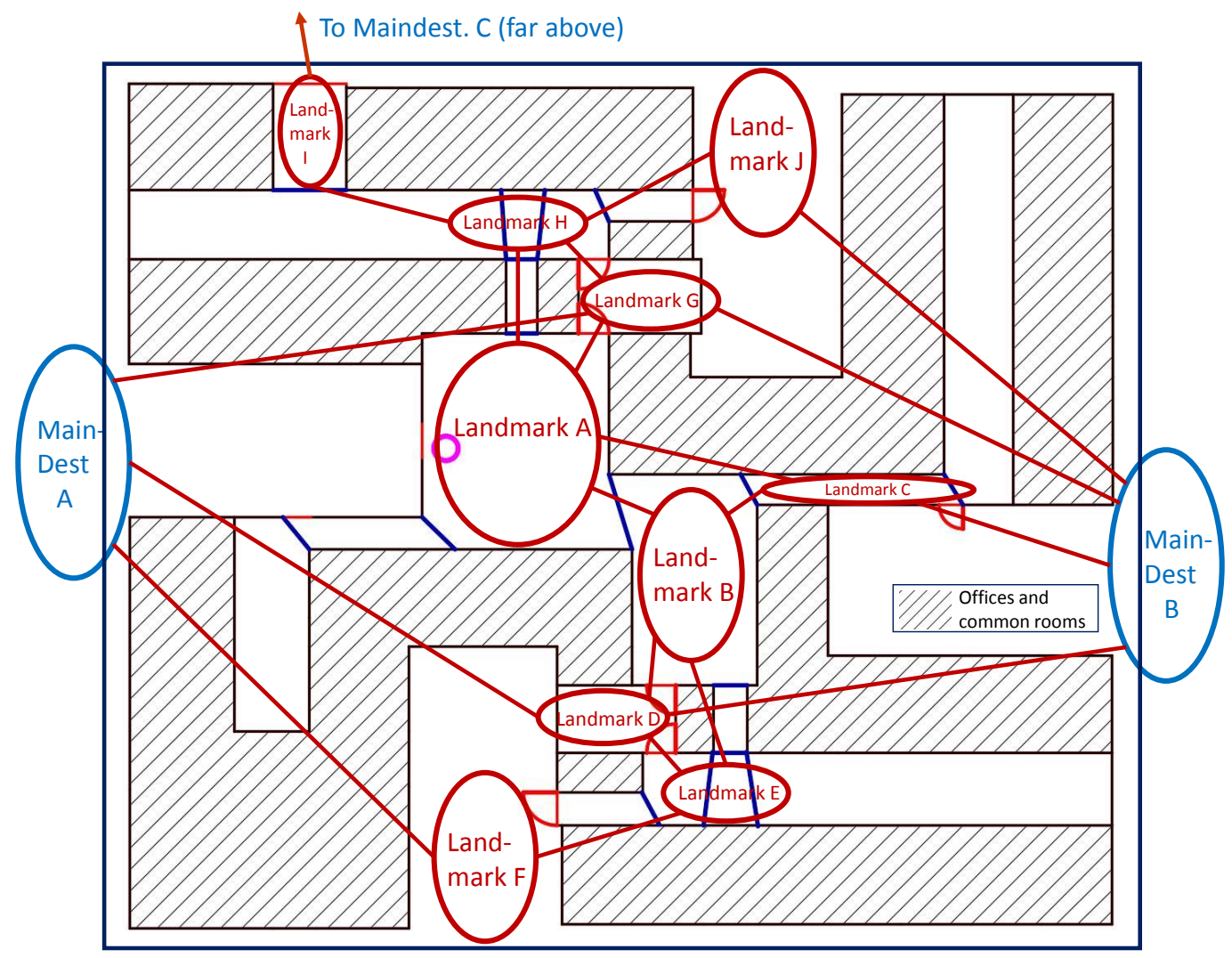

Figure 14. In the background: The floor plan of the area which was used for the simulation cases. Blue lines depict crossings between rooms (or edges in the underlying graph network). Red lines depict final exits. In the foreground: Landmarks and main destinations that are incorporated in a cognitive map that represents comprehensive spatial knowledge.

To create cognitive maps for groups of agents with different knowledge degrees, a specific proportion of landmarks and/or main destinations are randomly removed from the map. 
The remaining question is how many percent of landmarks have to be removed to describe the knowledge degrees of specific groups of subjects appropriately (for example the group of persons that rated their knowledge with the value 8 ). To address this question, we introduce a function to describe the relation between the group's knowledge degree $k$ and the percentage of randomly removed landmarks. In this case, the proportion of randomly removed landmarks equals the probability $P(k) \in[0,1]$ that a certain landmark is removed. The probability $P(k)$ applies for all landmarks, thus all landmarks are treated equally. For the sake of simplicity, we use the linear function

$$
P(k)=m \cdot k+P_{0} ; \quad m, P_{0} \in \mathbb{R} .
$$

To find the pair of parameters $m$ and $P_{0}$ which is most appropriate to describe the relation between self-rated knowledge degree and part of removed landmarks, we proceed as follows: 470 simulations $^{9}$ of the field study's scenario for a specific pair of parameters are conducted. The simulations are carried out with the help of JuPedSim. In every simulation run, a single agent starts at the position from which also the subjects in the study started and tries to evacuate. The geometry set-up that is used here is shown in Fig. 14 .

Within a certain part of the 470 simulation runs for every parameter pair a single agent is equipped with a cognitive map that (described by the linear function) represents a specific knowledge degree. The relative share of runs with a certain knowledge degree corresponds to the relative part of subjects of the study with the respective knowledge degree. For example: 5 participants $(\approx 10.6 \%)$ rated their knowledge with the value 1. Thus, $10.6 \%$ of the runs (50 runs) are carried out using an agent provided with a cognitive map that describes the knowledge degree 1 . The agent's route is recorded in every run. Subsequently, the route distribution that resulted from the whole set of 470 simulation for a specific parameter pair is determined. In this context, the routes that have been considered in the evaluation of the field study are considered as well. The route distribution is then compared to the distribution that resulted from the field study and the percentile deviation of usage for every route is calculated. Finally, the average deviation of route usages is determined.

For the slope $m$ we choose the values $0.025,0.050, \cdots, 0.3$. We assume that values which are located beyond this interval do not lead to realistic route distributions as the slope is either too steep or too gentle to describe the influence of the knowledge degree appropriately. These values are each combined with 10 values for the parameter $P_{0}$ starting with $-7.0 \cdot m$ followed by $-7.0 \cdot m+\frac{1}{9}$ followed by $-7.0 \cdot m+\frac{2}{9}$ and so on. Hence, in total 120 parameter pairs are considered.

The pair of parameters that leads to the lowest average deviation $\overline{d e} v_{\min }$ of the route distribution is considered to be the best choice to describe the relation between knowledge degree and omission of landmarks (see Eq. 4).

$$
\overline{d e} v_{\min }=\underset{m, P_{0}}{\arg \min } \operatorname{de} v\left(m, P_{0}\right) \text {. }
$$

After the execution of the $120 \cdot 470$ simulations, the pair consisting of the parameters $m=0.25$ and $P_{0}=-1.19444$ turned out to be the best choice. The comparison of the route usages are shown in Tab. 3 .

\footnotetext{
${ }^{9}$ Here we use a multiple of 47 (47 subjects) to obtain more data from the simulations and to rule out route usages that result by coincidence
} 
Table 3. Comparison of route usage that resulted from the field study and from simulations considering various route choice models. CMap $=$ The here presented cognitive map approach, $\mathrm{SP}=$ Shortest Path, Rd. $=\mathrm{Random}$ choice model, $\mathrm{NDH}=$ Nearest door heuristic. Values in the table describe the proportion of participants or rather agents that used a specific route. Values are given in percent.

\begin{tabular}{|c||c||c|c||c|c||c|c||c|c|}
\hline & Study & CMap & Dev. & SP & Dev. & Rd. & Dev. & NDH & Dev. \\
\hline Route A & 27.66 & 31.06 & 3.4 & 0.0 & 27.66 & 24.66 & 3.0 & 0.0 & 27.66 \\
\hline Route B & 23.4 & 5.32 & 18.09 & 0.0 & 23.4 & 8.72 & 14.68 & 0.0 & 23.4 \\
\hline Route C & 34.04 & 36.17 & 2.13 & 100 & 65.96 & 8.09 & 25.95 & 0.0 & 34.04 \\
\hline Route D & 4.26 & 0.85 & 3.4 & 0.0 & 4.26 & 2.06 & 2.2 & 0.0 & 4.26 \\
\hline Route E & 0.0 & 0.85 & 0.85 & 0.0 & 0.0 & 2.21 & 2.21 & 0.0 & 0.0 \\
\hline Route F & 0.0 & 0.64 & 0.64 & 0.0 & 0.0 & 1.89 & 1.89 & 0.0 & 0.0 \\
\hline Route G & 2.13 & 1.7 & 0.43 & 0.0 & 2.13 & 2.41 & 0.28 & 0.0 & 2.13 \\
\hline Route H & 0.0 & 1.91 & 1.91 & 0.0 & 0.0 & 6.39 & 6.39 & 0.0 & 0.0 \\
\hline Route I & 0.0 & 1.91 & 1.91 & 0.0 & 0.0 & 5.96 & 5.96 & 0.0 & 0.0 \\
\hline Route J & 2.13 & 2.13 & 0.0 & 0.0 & 2.13 & 6.1 & 3.97 & 0.0 & 2.13 \\
\hline Route K & 4.26 & 7.66 & 3.4 & 0.0 & 4.26 & 6.45 & 2.19 & 0.0 & 4.26 \\
\hline Route L & 2.13 & 9.79 & 7.66 & 0.0 & 2.13 & 25.06 & 22.93 & 100 & 97.87 \\
\hline \hline Avg. Dev. & - & & 3.65 & & 10.99 & & 7.64 & & 16.31 \\
\hline
\end{tabular}

We also compare route distributions that result from simulation runs using other route choice models. In this context, a shortest path algorithm (SP), the nearest door heuristic (NDH) and randomly made decisions (Rd.) are selected.

The random decision model provides that at every decision point (see Fig. 12) the probability that a specific way to proceed is selected is uniformly distributed. The route usage for the choice model can be calculated by hand considering a decision tree. We determined the route usage by simulations but use the hand calculation for verification purposes. The random choice model does not consider varying knowledge degrees. Every agent possesses no spatial knowledge at all. Hence, the model underestimates the usage of Route A and especially Route B and Route C. Particularly, these routes were chosen by familiar subjects in the study. The usage of Route L is overestimated. Participants of the study avoided to proceed via the left corridor next to the blocked balconies $\mathrm{E}$ and $\mathrm{F}$ as they were told that these balconies were blocked. The average deviation from the usage of the field study is about $7.66 \%$.

$\mathrm{SP}$ and NDH are deterministic approaches. As the agent always starts at the same position in each of the simulation runs, it chooses always the same route when considering one of these approaches. Route C describes the shortest path, thus it's taken by $100 \%$ of agents when using a shortest path algorithm. In consequence, the usage of Route $\mathrm{C}$ is overestimated and the usage of every other route is underestimated as long as the route has been chosen at least once in the study.

Under consideration of the NDH the agent will head to the left corridor in each run as the related crossing is located closest. Hence, Route L is chosen by $100 \%$ of the agents.

The deviations of the usages of both the SP (10.99\%) and the NDH (16.31\%) are even greater compared to the deviation of the random choice model. This is caused by the fact that SP and NDH do not imply any variations according to agents at all.

The here presented cognitive map approach does consider variations of spatial knowledge degrees between persons. Consequently, it reproduces the route usage with a smaller error compared to all other listed route choice models. Especially the usage of Route A and Route $\mathrm{C}$ are reproduced with a lower error (under $4 \%$ ) compared to other route choice models. Only the predicted usages of Route B (underestimated by $18.09 \%$ ) and Route L (overestimated by $7.66 \%$ ) deviate with an error larger than $(4 \%)$.

The deviation of Route L might be caused by the fact that the subjects were told about 
the blocked Balconies $\mathrm{E}$ and $\mathrm{F}$ and thus avoided paths that lead into the direction of these balconies. This influence that has been exerted by the experimenters is obviously not considered in the model. Thus, effects caused by such influences certainly cannot be reproduced. The overestimation of the usage of Route B might be caused by one or both of the following thoughts. On the one hand, a relatively high number of the unfamiliar subjects took Route B despite their lack of spatial knowledge. This number is higher than the number that would result when the paths are randomly chosen. Possibly, these subjects preferred to go to the other foyer due to its size or due to the brightness of its adjacent corridor. Attractions caused by the size or lighting conditions of rooms are not considered and thus cannot be reproduced either. On the other hand, the part of familiar participants that took Route B in the study is underestimated by the model as well. This is caused by the fact that agents that know both Route A and Route B always choose Route A due to its shorter length. The estimation of path lengths is not absolutely accurate. Randomly chosen points within the ellipses are used to calculate the lengths of paths. Nevertheless, it is obviously accurate enough so that Route A is favored over Route B in every case. In contrast, the subjects of the study might not be able to evaluate the path length with the necessary precision. Moreover, they possibly even did not try to evaluate it, but simply used the first known route that they remembered. The relative usage of A and B may change if size and shape of the ellipses that represent the position of the landmarks are varied. Consequently, the calculation of path lengths would become more inaccurate.

The average deviation from the route usage of the field study is about $3.65 \%$ when using the linear function $P(k)=0.25 \cdot k-1.19444$ to describe the relation between the agents' knowledge degrees and the proportion of preserved landmarks (see Fig. 15(a)). The course shows that agents which possess knowledge degrees lower than 5 are not provided with any landmarks at all. Cognitive maps of agents with a knowledge degree higher than 8 are comprehensive and contain all relevant landmarks. Remaining agents are provided with partially filled cognitive maps. The part of preserved landmarks can be read from the course of the linear function.

\section{Priority for specific landmarks}

The function only determines the proportion of preserved landmarks but does not give information about the question which landmarks are supposed to be incorporated in this part. In the above shown calibration the selection of kept landmarks has been performed randomly. Another reasonable procedure comprises the introduction of a priority for certain landmarks. For instance, it can be reasonable to privilege landmarks that are more salient than others or are located on paths that are more frequently used than other routes.

We carried out the above explained calibration approach again prioritizing the landmarks A, B, D, G and main destinations A and B. These landmarks are located on paths that are most frequently used by workers to get to their workplaces. In this context, the mentioned landmarks and destinations are selected to be kept by a probability that is twice as high compared to other landmarks or destinations. Under consideration of these privileges the use of the linear function with the parameter pair $m=0.3 P_{0}=-1.6555$ leads to the smallest average deviation of route usages in comparison to the results of the field study. In Fig. 15(b) the relation between knowledge degree and probability to keep a certain non-privileged landmark is shown by the blue line. The relation between knowledge degree and probability to keep a privileged landmark is depicted by the green dashed line. The average deviation is about $3.794 \%$, thus higher than the average deviation that resulted from the first calibration approach that does not consider any favoritism of certain landmarks. 


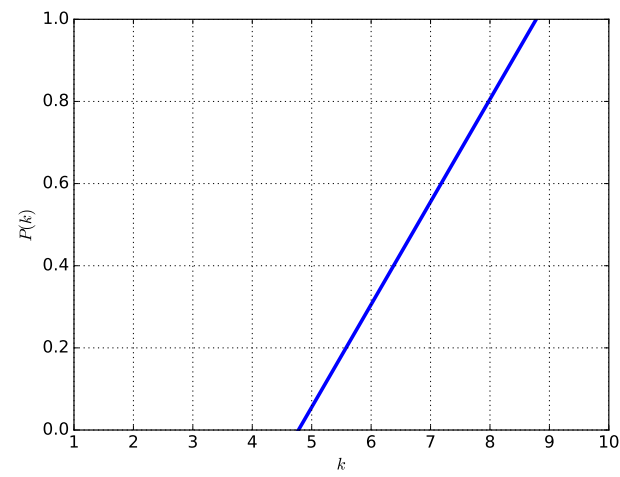

(a)

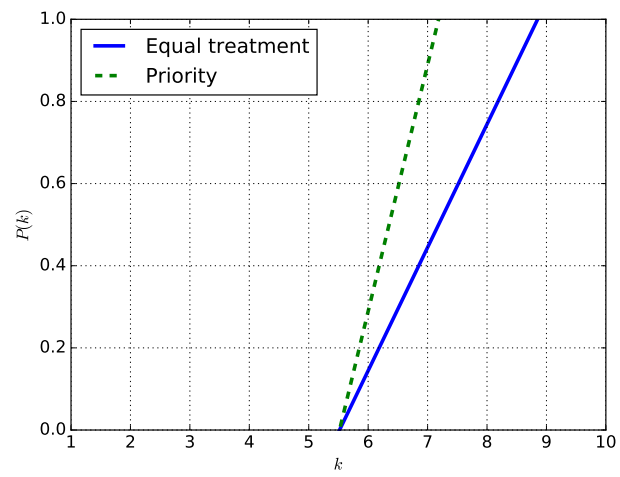

(b)

Figure 15. Relation between knowledge degree $k$ of an agent and probability $P(k)$ that a landmark or main destination is incorporated in its cognitive map. a) All landmarks and main destination are treated equally. b) The probability (green dashed line) that landmarks A, B, D, G and main destinations A and B are preserved is twice as high in comparison to the probability of other landmarks or main destinations (blue line).

\section{Discussion - Limits and possibilities of the proposed model}

With the help of the approaches to model partial and inaccurate knowledge that have been introduced in this paper we tried to reproduce the route usage that resulted from the field study. For this purpose, we created different cognitive maps to model spatial representations of various knowledge degrees. These maps have been established by removing a certain part of landmarks and destinations from a comprehensive map. A comparison showed that the route usage predicted by the calibrated modeling approaches are in good agreement with the route usage of the study. The average deviation is about $3.65 \%$ and thus remarkably lower than deviations that appear when using random route choices, the nearest door heuristic or a shortest path algorithm. The good agreement is mainly caused by the fact that our modeling approaches consider the existence of partial spatial knowledge. However, the usage of one route is seriously underestimated. This is partially caused by the fact that the model does not consider attractions by environmental factors. These could have been the reason why a relatively high amount of participants took the mentioned route.

It has been shown, that the model is a useful tool to reproduce the route choice distribution of the here presented field study. However, a generic applicability of the model to predict route choices in arbitrary buildings has not been tested yet and is therefore not guaranteed. In addition, it is not assured that the model is able to predict route choices of pedestrian groups that are mainly unfamiliar with the facility, for example first time visitors.

With the use of shortest path or travel time optimization algorithms the knowledge degree has been fixed to be comprehensive by the model itself. Now, with the use of the approaches presented here the user is responsible for the selection of appropriate knowledge degrees of every single agent. Therefore, the user can set-up various cognitive maps which differ from each other. In this context, the user has to decide which landmarks shall be incorporated in an individual map and has to select their shapes, sizes, and positions.

Unfortunately, it can be difficult to evaluate the spatial knowledge degrees of the pedestrians and thus find the correct input for the model. Every conceivable model that considers spatial knowledge thoroughly is accompanied by this issue. We suggest two possible ways to face this problem. Firstly, the conduction of a study, questionnaire, survey, etc. to determine how many (and how comprehensively) people are familiar with 
the facility/ how many are first time visitors. If this is not possible, for example since the facility has not been built yet, there is the option to make assumptions based on experiences/data from similar buildings or rather buildings of the same type.

Although the approaches consider several wayfinding aspects, they do not regard the influence of signage. Especially, first-time visitors or people who are very unfamiliar with the environment tend to rely on signs instead on their cognitive map as it is merely filled with information. Furthermore, the herding phenomena is not incorporated in the modeling framework. Particularly, concerning collective pedestrian dynamics in evacuation scenarios the herding effect could play a major role and has to be regarded. Nevertheless, spatial memories are still important and can have an impact on route choices even in facilities that possess a sufficient and well visible signage. That results from the fact that in certain circumstances people ignore the signage and prefer to use the route they came in or a route they are familiar with (?).

The modeling framework comprises a spatial learning concept. In the context of this concept, the agents are capable to re-recognized rooms that have been visited during the simulation. We do not claim this concept to represent accurately and comprehensively human spatial learning processes. Instead, the learning concept has been created and implemented to make sure that agents do not "oscillate" back and forth between rooms or move in circles, so that they are able to implement their strategies expediently and find an exit eventually.

Perceptional abilities considered in this model are restricted, simplified, and generalized and do not nearly cover human abilities to perceive visible surroundings. Particularly, agents cannot consider information that lies beyond doorways of their actual rooms even if the crossings represent open doors or rather would not hide the visible area beyond them in reality.

The here presented model certainly does not cover human abilities involved in wayfinding comprehensively. It only models selected aspects in a generalized way. Besides, the representations of many parts that were partly developed under applied assumptions are simplifications of the reality. Nevertheless, the modeling approaches provide advantages, for example the consideration of partial spatial knowledge in collaboration with generalized experiences, in comparison to state-of-the-art route choice models.

\section{Conclusion \& Outlook}

In order to improve the reliability of evacuation simulations in complex buildings, we introduce in this work modeling approaches of selected wayfinding abilities. Particularly, we propose a representation of inaccurate partial spatial knowledge (the cognitive map). The model enables agents to make an intelligent route choice decisions based on their individual cognitive map and also in consideration of generalized knowledge and spatial memories they gathered during their evacuation.

With the help of these modeling approaches it is even possible to provide simulated agents with distorted or wrong information about the environment beyond their field of view. The creation of various cognitive maps is possible by adding or omitting landmarks and/or connections between them. Furthermore, the information's inaccuracy about the items in the map can be varied by changing size, shape and position of the ellipses which represent the landmarks or rather their positions. Various compositions of landmarks and possible connections between them are conceivable covering representations of point, route and survey knowledge.

The model incorporates an algorithm which evaluates the actual cognitive map and determines the landmark most appropriately serving as the next subdestination. The 
path finding algorithm will find the most expedient (under consideration of the actual knowledge degree) doorway to reach the subdestination even if it's not located in the adjacent room. Additionally, exit choices are also made under the consideration of generalized knowledge dealing with general properties of the type of a building. Moreover, the integration of further factors that regard congestion in front of doors (?) and/or smoke propagation (?) for example is conceivable.

Additionally, in this paper, we present the results of a field study we conducted in an office building. Purpose of this study was to investigate the participants' route choices in the building with respect to their knowledge degree. The field study has shown that route choices can be remarkably influenced by the familiarity of a building's users or visitors. Certain routes could be identified in the study that were particularly preferred by subjects who were familiar with the spatial structure. Almost all participants in possession of comprehensive knowledge about the building took the shortest path. However, although a majority of the participants work in the regarded building, only $\approx 20 \%$ claimed their knowledge to be profound (degree 9 or 10). A majority of the participants who work in the building but do not know or could not evaluate the shortest path took familiar routes e.g. routes they daily use to reach their workplace. Unfamiliar participants possibly chose their routes randomly or were attracted by brighter or greater corridors.

The study's results have been used to calibrate the cognitive map model. In this context, we showed that the calibrated cognitive map model is able to reproduce the study's route choice distribution with minor discrepancy. Nevertheless, a generic applicability of the model cannot be guaranteed yet. Still more empirical data is necessary to figure out whether the model provides reliable route choice predictions in arbitrary buildings and for arbitrary compositions of occupants.

The model can be utilized for the calculation of a range of multiple possible evacuation times by carrying out multiple simulations which incorporate the consideration of various collocations of knowledge degrees. In this context, it can be evaluated how remarkably the evacuation time can deviate in comparison to an egress time that results from a simulation that uses a shortest path algorithm (see ? as an example). Depending on the deviation the user is able to decide whether the application of shortest path algorithms is still acceptable in the context of his scenario (possibly because the spatial structure of the scenario is trivial).

With the help of the model buildings of various types can be assessed concerning route guidance, composition of landmarks, set-up and dimensions of doors, circulation and functional rooms. Especially the effects of certain disposals of salient or rather non-salient landmarks regarding route usages can be examined. This enables a building's planner to find an appropriate position to set an anchoring landmark which might increase the legibility of the building's structure.

Beside the advantages that are provided for buildings' design and evaluation purposes the modeling framework offers new possibilities for discussions about the wayfinding procedure itself. The model shows i.a. the effects of a wayfinding behavior which is influenced by both spatial representations and generalized knowledge.

For future work we plan to use visibility polygons (isovists) that determine the current area an agent can see. The use of isovists allows recognition of signs and other people. We plan to extend our approaches and include the consideration of signage and herding effects to obtain a modeling framework that comprises all relevant tools and strategies used in wayfinding tasks. 
